\title{
Planar Liquid-like Arrangement of Photopigment Molecules in Frog Retinal Receptor Disk Membranes
}

\author{
J. K. Blasie† and C. R. Worthington \\ Department of Physics and Biophysics Research Division \\ The University of Michigan, Ann Arbor, Mich., U.S.A.
}

(Received 8 May 1968, and in revised form 26 August 1968)

Low-angle $\mathrm{X}$-ray diffraction arising from 40 to $50 \AA$ particles within wet frog retinal receptor disk membranes at $26^{\circ} \mathrm{C}$ was not consistent with a planar crystalline lattice of the particles within the disk membranes. The nature of the diffraction suggested the possibility of a planar liquid-like arrangement of the particles. Such an arrangement is supported by the observation that the planar ordering of the particles is easily altered by their interaction with globular protein molecules non-specifically adsorbed to the disk membranes. In view of the above, we obtained diffraction patterns from our wet disk membrane preparations at several temperatures between 4.5 and $42.5^{\circ} \mathrm{C}$, and applied a Fourier analysis to the diffracted intensities appropriate for a planar liquid-like arrangement of the 40 to $50 \AA$ particles. The analysis gave the planar radial distribution function description of the supposed planar liquid-like arrangement of the particles. These radial distribution functions, derived from the diffracted intensities, were examined in terms of their shape and variation with temperature, and compared with the known predictions from statistical mechanics for a liquidlike arrangement of particles whose pair potential contains both attractive and repulsive terms. This comparison for the derived radial distribution functions demonstrated that the observed diffraction data from the 40 to $50 \AA$ particles were indeed consistent with a planar liquid-like arrangement of these particles within the disk membrane.

Our radial distribution function analysis allowed model scattering factors for the particles to be tested. It was found that only hard sphere cross-sectional electron densities for the particle with diameters of 40 to $44 \AA$ or reasonably hard, soft-sphere cross-sectional electron densities, with a core of uniform electron donsity 38 to $40 \AA$ in diameter and a total diameter of 44 to $46 \AA$, gave good agreement.

A similar analysis was applied to the diffracted intensities arising from the antirhodopsin molecules adsorbed to the wet disk membranes which had been treated with our antirhodopsin serum and is discussed relative to the preceding paper (Blasie, Worthington \& Dewey, 1969). A comparison of the radial distribution functions for the antirhodopsin molecules adsorbed to the antirhodopsin serum treated disk membranes and the 40 to $50 \AA$ particles of the untreated disk membranes at identical temperatures showed the particles to be the photopigment molecules.

The mathematical derivation of the planar radial distribution function and a critical evaluation of the errors involved are presented in the Appendices.

\section{Introduction}

In the previous paper (Blasie, Worthington \& Dewey, 1969), we describe low-angle $\mathrm{X}$-ray diffraction from particles 40 to $50 \AA$ in diameter within frog retinal receptor

$\dagger$ Present address: Biophysics Dept., University of Pennsylvania, Philadelphia, Pa., U.S.A. 
disk membranes. We demonstrated that these particles were the photopigment molecules of these disk membranes. However, inasmuch as the quantitative treatment of the low-angle $\mathrm{X}$-ray diffraction data was dependent on the calculations presented in this paper, we shall still refer to the photopigment molecules as 40 to $50 \AA$ particles of the receptor disk membranes and again prove that they are indeed the photopigment molecules.

We have shown (Blasie, Dewey, Blaurock \& Worthington, 1965; Blasie et al., 1969) that low-angle $\mathrm{X}$-ray diffraction from wet pellets of isolated disk membranes have cylindrical symmetry about an axis normal to the plane of the disk membranes. With the X-ray beam incident along this axis, we record diffraction information relating to electron density variation within the plane of the disk membranes. It is reasonable that such diffraction arises from the planar arrangement of the 40 to $50 \AA$ particles within the disk membranes (see footnote $\nmid$ p. 428 of this paper).

In the previous paper, we showed that non-specific adsorption of serum albumin to the disk membranes disrupted the ordering of the 40 to $50 \AA$ particles within the disk membranes. Thus, the ordering of these particles is easily altered by weak interaction with a foreign protein such as albumin. Table 1 of that paper shows that the two low-angle X-ray diffracted intensity maxima obtained from untreated wet disk membranes at $26 \cdot 0^{\circ} \mathrm{C}$ correspond to a Bragg spacing ratio $\left(d_{1} / d_{2}\right)$ of $1.52 \pm 0.01$. It is unlikely that a planar crystalline lattice of the 40 to $50 \AA$ particles could account for such a Bragg spacing ratio even if thermal motion of the particles about the lattice points occurred. Furthermore, the shape of the two intensity maxima, the first being moderately sharp compared to the second broad diffuse maxima, is rather characteristic of X-ray diffraction from a liquid-like arrangement of the particles (see for example James, 1948, chapter 9). This then suggests that these particles have a planar liquid-like arrangement within the receptor disk membranes.

The radial distribution function theory for liquids is well known from statistical mechanics (see Kirkwood, Lewinson \& Alder, 1952; Hill, 1956). This function and its temperature dependence has been characterized for particles interacting via repulsive potential functions only as well as potential functions containing both repulsive and attractive terms such as the Lennard-Jones "6-12" potential. Thus, we decided to explore the low-angle $X$-ray diffraction arising from the 40 to $50 \AA$ particles in the disk membranes over a reasonable temperature range, to calculate their radial distribution functions assuming a planar liquid-like ordering of these particles, and to test this hypothesis by comparison with the predictions of statistical mechanics for such a system. Such a test would involve the general shape and asymptotic behavior of the radial distribution functions, its temperature dependence, and its prediction of a four nearest neighbors for the crystalline state, i.e. the square: array of these 40 to $50 \AA$ particles occurring in partially dried receptor disk membranes. as established by electron microscopy and low-angle X-ray diffraction (Blasie et al., 1965). This paper describes the results of such an investigation.

\section{Materials and Methods}

A detailed account of the experimental methods and equipment used was presented in the previous paper (Blasie et al., 1969). For the present paper, the following description is given.

Specimens of frog (Rana pipiens) retinal receptor disk membranes and disk membranes treated with antirhodopsin sera were prepared in the same manner as described previously.

Low-angle X-ray diffraction patterns were obtained with the X-ray beam incident. 
normal to the planes of the disk membranes, i.e. along the sedimentation axis, in all cases. All exposure times were $2 \mathbf{h r}$ in duration and the disk membrane preparations remained fully hydrated, i.e. wet, with frog Ringer's solution for the duration of the exposure. The desired temperature of the specimen was maintained within $\pm 0.2 \mathrm{deg}$. $\mathrm{C}$ with the temperature chamber deseribed in the previous paper and the specimens were allowed to equilibrate for $30 \mathrm{~min}$ in the chamber before the 2 -hr exposure was begun. Low-angle patterns were obtained from untreated wet disk membranes at $4.5,26.0$ and $42.5^{\circ} \mathrm{C}$ and from wet disk membranes treated with antirhodopsin serum at $26.0^{\circ} \mathrm{C}$. Low-angle patterns were obtained from different untreated disk membrane preparations at different temperatures as well as from the same disk membrane preparation at temperatures in sequence with 2 -hr exposure times at each temperature with a 30 -min equilibration time between exposures. Such diffraction patterns at a given temperature showed no apparent differences with respect to the history of the specimen.

Microdensitometer tracings were made of all diffraction patterns in the range of $0.006 \leq r^{*} \leq 0.024 \AA^{-1}$. The error in intensity measurement was $\pm 5 \%$ judging from the noise level on the microdensitometer trace. Background scattering for this camera and these specimens was known over the same angular range and was subtracted from the tracing obtained with the receptor disk mernbrunes.

The low-angle data was recorded using a narrow X-ray beam of small finite height $(1 \mathrm{~mm})$. A discussion of possible smearing effects due to the finite height of the X-ray beam is given. Because our effective slit height was only $1 \mathrm{~mm}$ any slit correction is small and hence the corrected intensity can be expanded in terms of the uncorrected intensity and its derivatives (see Guinier \& Fournet, 1955, p. 119). By substituting the parameters of our camera (height of beam $1 \mathrm{~mm}$; specimen-to-film about $6 \mathrm{~cm}$ ) into this expression only the first derivative needs to be evaluated because, within the observed angular range, the coefficients of higher derivatives are vanishingly small. The maximum slit correction occurs on the upward slope of the first peak $\left(r^{*}=0.01 \AA^{-1}\right)$; this correction is $\$ 3 \%$ of the diffracted intensity at $r^{*}=0.01 \AA^{-1}$. For comparison the slit correction on the downward slope of the second peak $r^{*}=0.02 \AA^{-1}$ is $\$ 1.5 \%$. The correction is zero at the minima and maxima of the diffraction patterns. Hence any slit correction did not exceed $3 \%$ and, as this magnitude was within the range of experimental error in recording the intensities, slit corrections were not applied.

The diffracted intensity curves corrected for background were used to calculate respectively the radial distribution functions for the 40 to $50 \AA$ particles of the receptor disk membranes at the various temperatures mentioned and for the antirhodopsin molecules adsorbed to antirhodopsin serum treated disk membranes. The diffraction theory on which the analysis is based will be presented in the section Fourier Analysis of the Diffracted Intensities with references to the Appendices. All non-trivial integrations and computations were performed on an IBM 7090 computer.

\section{Results}

The low-angle X-ray diffraction patterns obtained from wet receptor disk membranes at $4 \cdot 5,26 \cdot 0$ and $42 \cdot 5^{\circ} \mathrm{C}$ are shown on Plate I. Figure 1 shows their respective microdensitometer tracings. We note that the first intensity maxima remains relatively constant in position, shape, and magnitude while the second diffuse maxima broadens, becomes less intense, and shifts its position to larger $r^{*}$ values $\left(r^{*}\right.$ is the reciprocal space distance where $r^{*}\left(\AA^{-1}\right)=\frac{(2 \sin \theta)}{\lambda}=\frac{1}{d(\AA)}$ where $d(\AA)$ is the Bragg spacing) with increasing temperature. Plate II and Figure 2 shows the low-angle pattern obtained from wet antirhodopsin serum treated disk membranes at $26 \cdot 0^{\circ} \mathrm{C}$ and its microdensitometer tracing. We note the positions of the intensity maxima in reciprocal space are nearly identical to those for untreated wet disk membranes at $26 \cdot 0^{\circ} \mathrm{C}$ although the diffracted intensity maxima are much sharper and more intense for the former. 


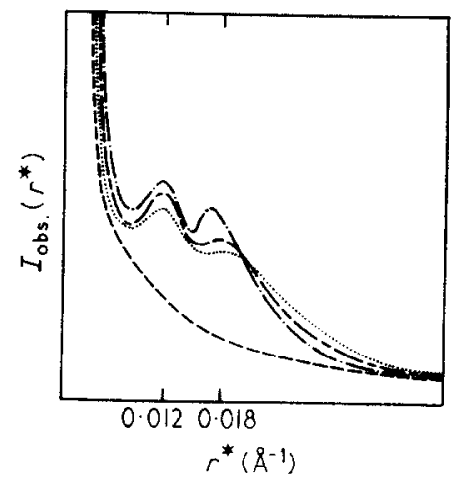

Fra. 1. Miorodensitometer tracings of the low-angle X-ray diffraction patterns shown in Plate $I$. The observed relative intensity $I_{\text {obs }}\left(r^{*}\right)$ is plotted as a function of the reciprocal space distance $r^{*}$. $\ldots . ., 42.5^{\circ} \pm 0.2$ deg. $\mathrm{C} ;--\longrightarrow, 26.0^{\circ} \pm 0.2 \mathrm{deg} . \mathrm{C} ;-., 04.5^{\circ} \pm 0.2 \mathrm{deg} . \mathrm{C}$.

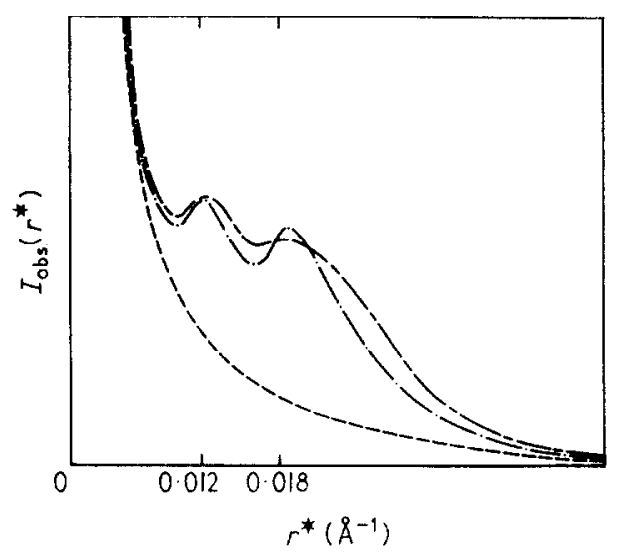

Fra. 2. Microdensitometer tracing of Plate II (antirhodopsin serum treated disk membranes at $26.0^{\circ} \pm 0.2 \mathrm{deg} . \mathrm{C}$ ) compared with that of Plate $\mathrm{I}(\mathrm{b})$ (untreated disk membranes at $26.0^{\circ} \pm$ $0.2 \mathrm{deg} . \mathrm{C})$.

- - Untreated, $26^{\circ} \pm 0 \cdot 2$ deg. $\mathrm{C} ;-.-$, antirhodopsin serum treated, $26^{\circ} \pm 0 \cdot 2 \mathrm{dog} . \mathrm{C}$.

\section{Preliminary Discussion of the Results}

The nature of the low-angle X-ray diffraction arising from the 40 to $50 \AA$ particles within wet receptor disk membranes as well as its temperature behavior is not characteristic of a planar crystalline lattice arrangement of the particles within the membranes. Over the temperature range investigated the ratio of the Bragg spacings for the two intensity maxima observed at any given temperature was not representative of a planar crystalline array of these particles within the disk membranes. Furthermore, the position (in reciprocal space) and the shape of the second intensity maxima changed as a continuous function of temperature while the first intensity maxima remained relatively constant in position and shape. The effect of temperature on a crystalline lattice, i.e. the effect of isotropic thermal motion of particles about their respective lattice points, does not shift the relative positions of the diffracted intensity maxima in reciprocal space. It merely multiplies the diffracted intensity 


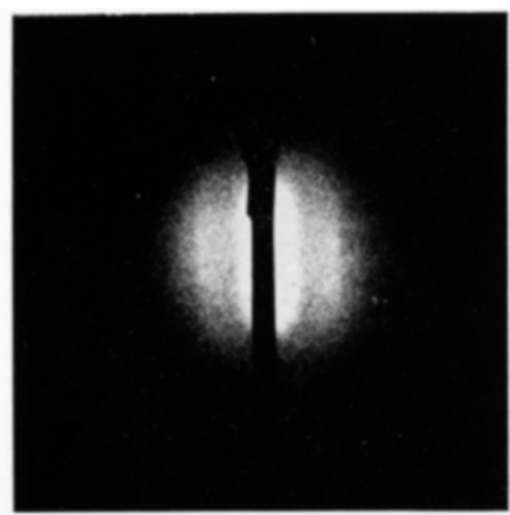

(a)

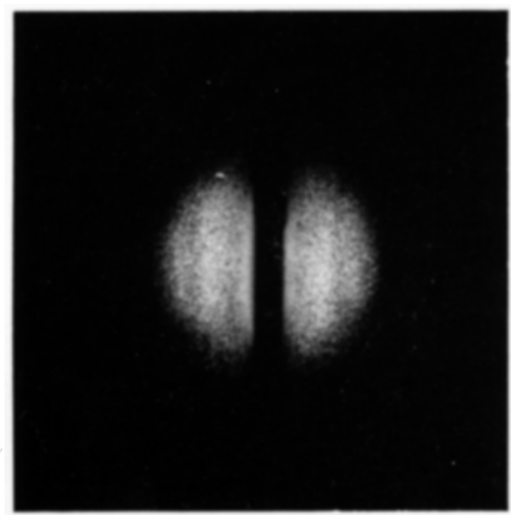

(c)

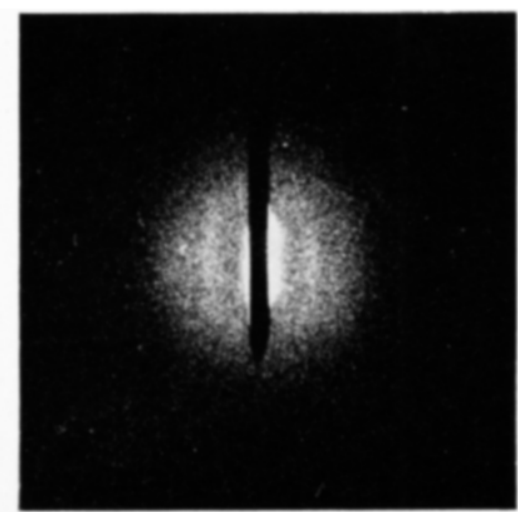

(b)

Plate I

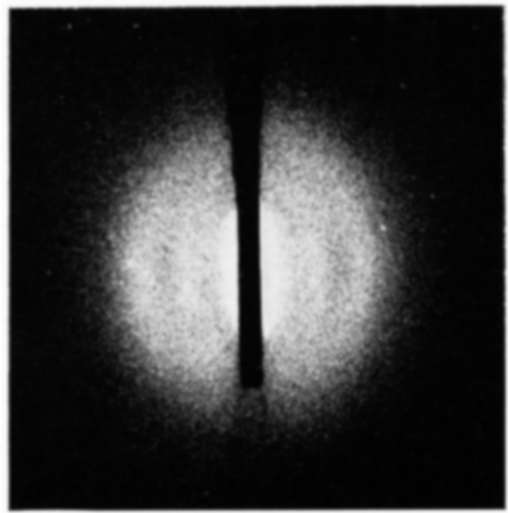

Plate II

Plate I. Low angle X-ray diffraction patterns obtained from completely wet ultracentrifugal pellets of frog retinal receptor disk membranes. The beam incident was along the sedimentation axis, that is, normal to the planes of the disk membranes as verified in the previous paper (Blasie et $a l,, 1969$ ). Exposures were $2 \mathrm{hr}$ in duration and were obtained at the following temperatures: (a) $42 \cdot 5^{\circ} \pm 0 \cdot 2$ deg. $\mathrm{C}$; (b) $26 \cdot 0^{\circ}+0 \cdot 2$ deg. $\mathrm{C}$; (c) $4 \cdot 5^{\circ} \pm 0 \cdot 2$ deg.C.

Plate II. Low-angle $\mathrm{X}$-ray diffraction pattern obtained from a completely wet ultracentrifugal pellet of frog retinal receptor disk membranes which had been treated with our antirhodopsin serum before sedimentation. The incident beam was normal to the planes of the disk membranes and the exposure was $2 \mathrm{hr}$ in duration at $26 \cdot 0^{\circ} \pm 0 \cdot 2 \mathrm{deg} . \mathrm{C}$. 
maxima by a factor $\exp (-2 M)$ where $M$ is directly proportional to the square of $r^{*}$ and increases with temperature (see, for example, Guinier, 1963).

Such temperature dependent X-ray diffraction is characteristic of a liquid-like arrangement of the 40 to $50 \AA$ particles within the disk membranes. Inasmuch as the low-angle $\mathrm{X}$-ray diffraction from the wet disk membranes is cylindrically symmetric about an axis normal to the planes of the disk membranes, the observed diffraction is characteristic of a planar or two-dimensional liquid-like arrangement of the 40 to $50 \AA$ particles within the membranes. This is indeed reasonable since these particles are confined to the disk membrane. Since the position of the second intensity maxima shifts to larger reciprocal space distance values with increasing temperature while the position of the first intensity maxima remains unchanged, it is apparent, in a qualitative sense, that most probable center-to-center distances for the 40 to $50 \AA$ particles decrease with increasing temperature. For a planar liquid-like arrangement of the particles, such behavior would indicate that the area available to the particles was independent of temperature and hence the particles occur with a constant average density over the entire disk membrane. This is also reasonable since these particles are confined to the disk membrane and their population per disk membrane is presumably conservative with temperature variation.

In view of the above evidence, we decided to apply a Fourier analysis for a planar liquid-like arrangement of the 40 to $50 \AA$ particles to the low-angle $\mathrm{X}$-ray diffraction from the untreated wet receptor disk membranes obtained at several temperatures. Such a Fourier analysis would give the radial distribution function representation for the planar liquid-like arrangement of the particles and the statistical function along with its temperature dependence could be compared with the well-known predictions of the statistical mechanics for simple liquids.

For the purpose of the previous paper, a similar Fourier analysis was applied to the low-angle $\mathrm{X}$-ray diffraction arising from antirhodopsin molecules adsorbed to the surface of antirhodopsin serum treated disk membranes. The radial distribution function for the antirhodopsin molecules obtained from this analysis may then be compared with that of the 40 to $50 \AA$ particles of untreated disk membranes allowing a quantitative comparison of the planar liquid-like arrangement of the adsorbed antirhodopsin molecules with that of the 40 to $50 \AA$ particles themselves.

\section{Predictions from the Statistical Mechanics of Simple Liquids}

Inasmuch as we have used the radial distribution function approach to the statistical mechanics of a planar liquid, we wish to discuss the properties and interpretation of this statistical function. The general properties of radial distribution functions and their temperature dependence are well known for real particles whose interaction potential contains both attractive and repulsive terms such as the Lennard-Jones potential. For a detailed account of these properties the reader is referred to Kirkwood et al. (1952) or Hill (1956). For the purpose of this paper, it will suffice to state these properties as follows: the radial distribution function for a particle in a two-dimensional or planar liquid is a function of the radial co-ordinate $r(\AA)$, is temperature dependent, and will be designated as $2 \pi r \rho_{p}(r)$; the function should ideally be zero for $r$ less than the particle diameter, i.e. the distance of closest approach for the particles; the radial distribution function has a first maxima (for $r$ greater than the particle diameter) whose amplitude is greater than $2 \pi r \rho_{0}$ where $\rho_{0}$ is the average density of particles over the disk membrane area; this first maxima 
is followed by successive oscillations of diminishing amplitude about $2 \pi r \rho_{0}$ such that $\lim _{r \rightarrow \infty} 2 \pi r \rho_{\mathrm{p}}(r)=2 \pi r \rho_{0}$. Such a distribution represents the time-averaged probability density of particles occurring within an annular ring in the plane of the disk membrane of radius $r$ and width $\mathrm{d} r$ contered about an arbitrary particle. The successive maxima of this function for $r$ greater than the particle diameter, are denoted the first, second, etc. neighbor peaks with reference to the origin and the area under these peaks represents respectively the time-averaged number of first, second, etc. nearest neighbors for any arbitrary particle in the plane of the disk membrane. The $n$th nearest neighbors occur within an annular ring in the plane of the mem. brane centered about an arbitrary particle whose radius is that of the $n$th maxima in the radial distribution function and whose half-width is the half-width of the $n$th maxima.

With increasing temperature, the nearest neighbor peaks become broader and of lesser amplitude and shift toward the origin provided that a constant average density of particles is maintained. 'The first nearest neighbor peak approaches the particle diameter or distance of closest approach for the particles and the number of first nearest neighbors decreases. With decreasing temperature, the nearest neighbor peaks become sharper and have greater amplitude and shift away from the origin such that position of the first nearest neighbor peak approaches the lattice constant of the crystalline state and the number of first nearest neighbors approaches that of the crystalline state.

Having discussed the properties and temperature behavior of radial distribution functions describing a planar liquid-like arrangement of particles as predicted from statistical mechanics for particles interacting via a "real" interaction potential in a system of constant average particle density over the plane, we wish to discuss the Fourier analysis of the diffracted intensity from the 40 to $50 \AA$ particles of the disk membranes at various temperatures which gives the experimentally determined radial distribution functions for the particles at those temperatures.

\section{Fourier Analysis of the Diffracted Intensities}

The radial distribution function analysis has been used in the study of spherically symmetric liquid and amorphous systems composed of atoms and small molecules. The radial distribution function representation for such systems is obtained by applying the Fourier integral theorem to the X-ray diffraction intensities (James, 1948). The radial distribution function approach applies equally well to macromolecular systems but seems not to have been previously used. Most X-ray studies on macromolecular systems are confined to dilute aqueous solutions of one macromolecular species so that intermolocular effects and solvent struoture can be neglected; these studies only give macromolecular shape. It is appropriate to apply the radial distribution function analysis to our macromolecular system as the packing of the 40 to $50 \AA$ particles in the disk membrane is sufficiently dense to show diffraction due to intermolecular effects. The Fourier analysis (as applied to simple liquids) needs modification as we are studying a planar arrangement of these densely packed macromolecules in a complex system, namely a biological membrane, containing additional macromolecular species. The $\mathrm{X}$-ray diffraction treatment is given in the Appendices and references concerning related analytical procedures as applied to atomic and small molecule liquids are given there. An important consequence of 
using such an analysis is that we are able to make a study of macromolecular shape directly from our radial distribution function curves.

The radial distribution function $2 \pi r \rho_{\mathrm{p}}(r)$ describing a planar liquid-like arrangement of particles in the disk membranes is given in terms of the observed diffracted intensity $I_{\text {obs }} .\left(r^{*}\right)$ as follows (see Appendix 1 for details):

$$
2 \pi r \rho_{\mathrm{p}}(r)=2 \pi r \rho_{\mathrm{o}}+2 \pi r \int_{0}^{\infty} 2 \pi r^{*}\left\{\frac{K I_{\mathrm{obs}}\left(r^{*}\right)}{N f_{\mathrm{p}}^{2}\left(r^{*}\right)}-1\right\} J_{0}\left(2 \pi r r^{*}\right) \mathrm{d} r^{*} .
$$

$I_{\text {obs. }}\left(r^{*}\right)$ is the observed intensity (on a relative scale) cylindrically symmetric about an axis normal to the plane of the disk membrane, i.e. the sedimentation axis, with $r^{*}$ as defined in the Results section. $I_{\text {obs. }}\left(r^{*}\right)$ is brought to an absolute scale via the normalization constant $K$ (see Appendix 2 for intensity normalization methods). $f_{\mathrm{p}}\left(r^{*}\right)$ is the scattering factor for each of the $N$ particles composing the planar liquid (see Appendix 4 for the determination of the particle scattering factors).

However, in practice one does not observe diffracted intensity over all of reciprocal space and the integral in equation (1) is terminated at some upper limit $r_{0}^{*}$ introducing transform truncation errors in the derived radial distribution function (see Appendix 3 for a determination of such errors).

Assuming that the number of 40 to $50 \AA$ particles per disk membrane is conserved upon partially drying the disk membranes, $\rho_{\mathrm{u}}$ may be determined knowing that the particles occur in a square array in partially dried disk membranes with one particle per unit cell, i.e. per $4900 \AA^{2}$ of disk membrane area.

Scattering factors were calculated for the 40 to $50 \AA$ particle of the untreated disk membranes and for the cylindrical antirhodopsin molecule adsorbed to the antirhodopsin serum treated disk membranes according to the methods discussed in Appendix 4. The radial distribution functions were then calculated for the particles of the untreated wet disk membranes using the normalized intensity data obtained at $4.5,26.0$ and $42.5^{\circ} \mathrm{C}$ as well as for the antirhodopsin molecules adsorbed to the serum treated disk membranes using the normalized intensity data at $26.0^{\circ} \mathrm{C}$ for this case. The integral in equation (1) was terminated when $K I\left(r^{*}\right) \rightarrow N f_{\mathrm{p}}^{2}\left(r^{*}\right)$ for $r^{*} \geqslant r_{0}^{*}$ (see Appendix 3) and was integrated numerically with an IBM 7090 using Simpson's three point rule sampling the normalized intensities at intervals of $\Delta r^{*}=$ $0.001 \AA^{-1}$. The results of such calculations are shown in Figures 3 and 4 . The calculation of nearest neighbor numbers was carried out via a numerical integration (Simpson's three-point rule with an IBM 7090) of the radial distribution function over the interval of the radial co-ordinate $r$ containing the nearest neighbor peak concerned (these nearest neighbor peaks were extrapolated to the abscissa such that the sum of the nearest neighbor peaks gave the radial distribution function). The results are shown in Table 1.

\section{Discussion of the Fourier Analysis}

It is apparent from Figure 3 and the section Predictions from the Statistical Mechanics of Simple Liquids that the properties and temperature behavior of the derived radial distribution functions for the 40 to $50 \AA$ particles of the disk membranes are in excellent accord with those predicted from statistical mechanics. All of the derived functions go to $2 \pi r \rho_{0}$ for large $r$ in the proper manner, contain the required first maxima for $r$ greater than the particle diameter (i.e. the first nearest 


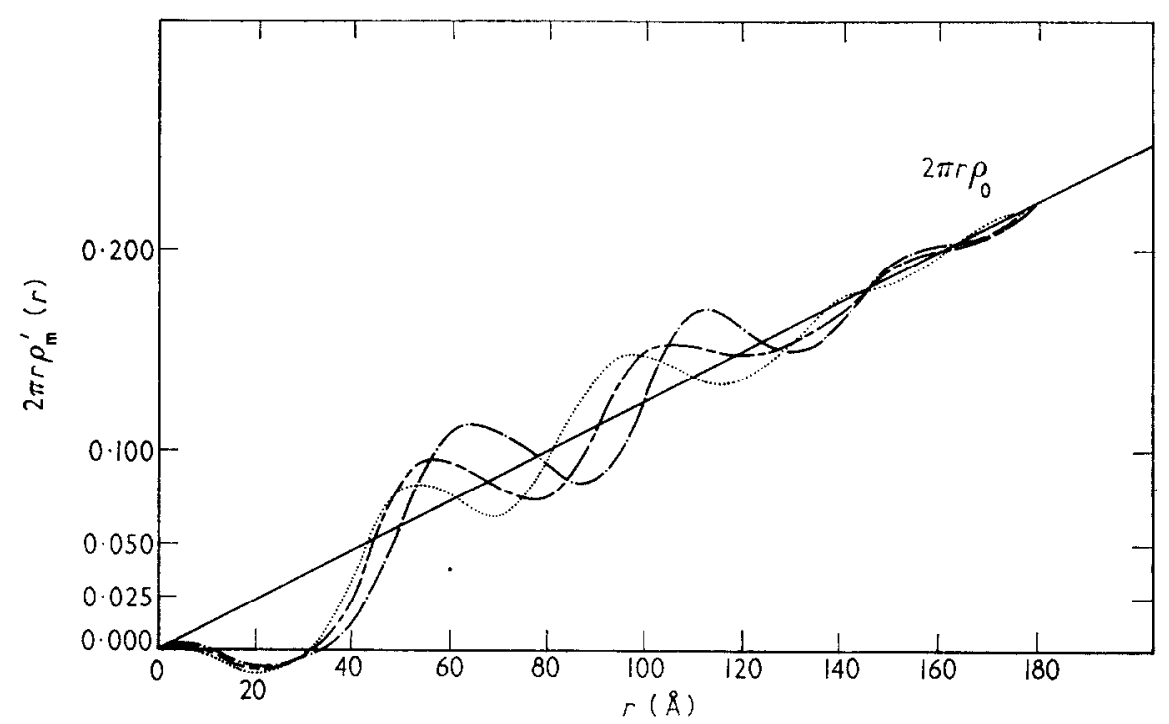

FrG. 3. Planar radial distribution functions for the particles of the wet untreated disk membranes at $4.5^{\circ} \pm 0.2 \mathrm{deg} . \mathrm{C}, 26.0^{\circ} \pm 0.2 \mathrm{deg} . \mathrm{C}$ and $42.5^{\circ} \pm 0.2 \mathrm{deg}$. C. The radial distribution functions were calculated according to Appendices 1, 2, and 4 using the best model particle scattering factor as desoribed in Appendix 4.

$\ldots \ldots, 42 \cdot 5^{\circ} \pm 0.2 \mathrm{deg} . \mathrm{C} ;----, 26 \cdot 0^{\circ} \pm 0.2 \mathrm{deg} . \mathrm{C} ;-.-, 04 \cdot 5^{\circ} \pm 0.2 \mathrm{deg} . \mathrm{C}$.

neighbor peak), and have only small oscillations in the region of $r$ less than the particle diameter which can be accounted for mainly by transform truncation effects (see Appendix 3). Furthermore, the number of first nearest neighbors decreases and the position of the first nearest neighbor peak shifts toward the origin approaching the particle diameter with increasing temperature as seen in Table 1. The first nearest neighbor peak also broadens and decreases in amplitude with increasing temperature as seen in Figure 3. Conversely, the first nearest neighbor peak becomes sharper with greater amplitude, and shifts away from the origin with decreasing temperature. The position of the first nearest neighbor peak approaches the unit cell dimension of the crystalline state of $70 \AA$ and the number of first nearest neighbors approaches

\section{Table 1}

\begin{tabular}{|c|c|c|c|c|c|}
\hline & $\begin{array}{c}\text { Temp. } \\
\left({ }^{\circ} \mathrm{C}\right)\end{array}$ & $\begin{array}{l}\text { 1st Nearest } \\
\text { neighbor } \\
\text { number }\end{array}$ & $\begin{array}{c}\text { Time-average } \\
\text { separation of } \\
\text { lst nearest } \\
\text { neighbors }(\AA)\end{array}$ & $\begin{array}{c}\text { 2nd Nearest } \\
\text { neighbor } \\
\text { number }\end{array}$ & $\begin{array}{l}\text { Time-average } \\
\text { separation of } \\
\text { 2nd nearest } \\
\text { neighbors }(\AA)\end{array}$ \\
\hline $\begin{array}{l}40 \text { to } 50 \AA \text { Particles } \\
\text { of untreated wet } \\
\text { disk membranes }\end{array}$ & $\begin{array}{r}4 \cdot 5 \pm 0 \cdot 2 \\
26 \cdot 0 \pm 0 \cdot 2 \\
42 \cdot 5 \pm 0 \cdot 2\end{array}$ & $\begin{array}{l}3 \cdot 8 \\
3 \cdot 0 \\
2.5\end{array}$ & $\begin{array}{l}64 \cdot 0 \\
56 \cdot 0 \\
52 \cdot 0\end{array}$ & $\begin{array}{l}6 \cdot 5 \\
6 \cdot 1 \\
5 \cdot 8\end{array}$ & $\begin{array}{r}112 \cdot 0 \\
103 \cdot 0 \\
94 \cdot 0\end{array}$ \\
\hline $\begin{array}{l}\text { Antirhodopsin } \\
\text { molecules adsorbed } \\
\text { to antirhodopsin } \\
\text { serum treated wet } \\
\text { disk membranes }\end{array}$ & $26 \cdot 0 \pm 0 \cdot 2$ & $3 \cdot 1$ & $58 \cdot 0$ & 6.2 & $113 \cdot 0$ \\
\hline
\end{tabular}


the value of that of the crystalline state of 4.0 at a temperature slightly above freezing as seen in Table 1. Since the radial distribution functions for the 40 to $50 \AA$ particle of the retinal receptor disk membrane are of the proper character and temperature behavior as those predicted from statistical mechanics for particles interacting via an interaction potential containing both attractive and repulsive terms, we conclude that these 40 to $50 \AA$ particles do indeed occur in a planar liquidlike arrangement within the receptor disk membranes.

We should also like to point out that the radial distribution function is particularly sensitive to errors in the scattering factor for the particles in the region of small $r$ for $r$ less than the particle diameter. Thus, using the procedure and criteria discussed in Appendix 4, scattering factors appropriate for spherical lipid micelles were shown to be inconsistent with the diffracted intensity data since they invariably induced severe oscillations in the radial distribution functions for small $r$. The scattering factor most consistent with the diffracted intensity data was that appropriate for a globular protein molecule based on a reasonably hard "soft-sphere" cross-sectional electron density for the scattering particle with a core of uniform electron density 38 to $40 \AA$ in diameter and a total diameter of 44 to $46 \AA$.

With reference to Figure 4 and Table 1 it is apparent that the planar liquid-like arrangement of the antirhodopsin molecules adsorbed to the antirhodopsin serum treated disk membranes is nearly identical to that of the 44 to $46 \AA$ globular protein molecules of the untreated disk membranes at the same temperature. This follows

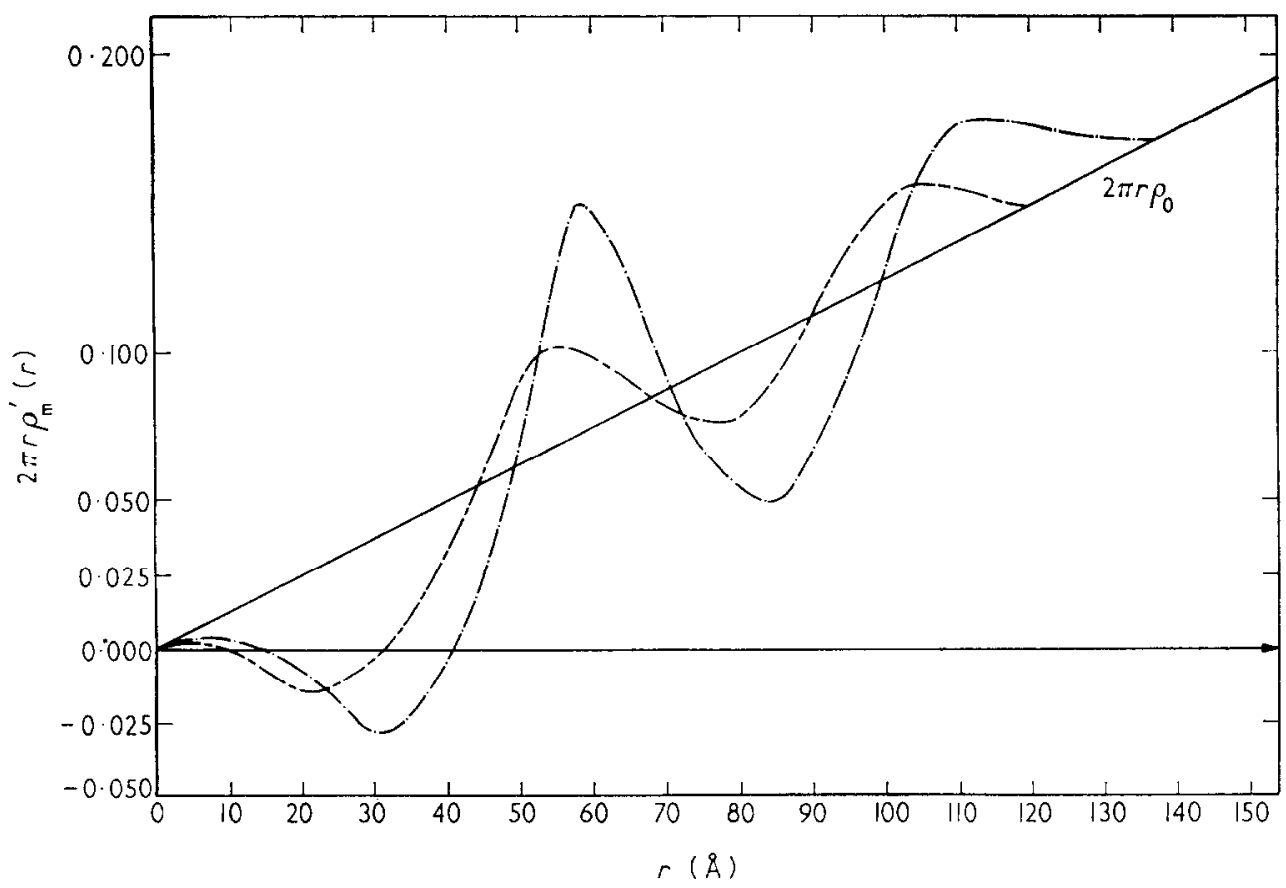

Fra. 4. Planar radial distribution function for the particles of the wet untreated disk membranes at $26 \cdot 0^{\circ} \pm 0.2 \mathrm{deg}$. C compared with that of the cylindrical antirhodopsin molecules adsorbed to the antirhodopsin serum treated disk membranes at $26 \cdot 0^{\circ} \pm 0 \cdot 2 \mathrm{deg}$. C. Again, best model particle scattering fuctors were used in both cases as described in Appendix 4.

- - - Untreated, $26^{\circ} \pm 0 \cdot 2 \mathrm{deg} . \mathrm{C} ;-\cdots$, , antirhodopsin serum treated, $26^{\circ} \pm 0 \cdot 2 \mathrm{deg}$. C. 
since the position of the first nearest neighbor peak and the number of first nearest neighbors are nearly identical for the two cases. Inasmuch as the antirhodopsin molecules have been shown to specifically bind the photopigment molecules of the retinal receptor disk membranes (Dewey, Blasie, Davis \& Barr, 1969; Blasie et al., 1969), we conclude that the 44 to $46 \AA$ globular protein molecules of the wet receptor disk membranes are indeed the photopigment molecules of these membranes.

In Appendix 4, we show that the best scattering factor for the oylindrical antirhodopsin molecule adsorbed to the surface of the antirhodopsin serum treated disk membranes is based on a "hard-cylinder" cross-sectional electron density for the molecule $32 \pm 1 \AA$ in diameter with the cylinder axis normal to the plane of the disk membrane. The nearest neighbor peaks of the radial distribution function for the antirhodopsin molecules adsorbed to serum treated disk membranes are considerably sharper and are shifted away from the origin slightly compared to those of the photopigment molecules alone. This is consistent with the following. The specific one-to-one binding of the cylindrical antirhodopsin molecules to the photopigment molecules in the disk membrane greatly increases the surface area available for nearest neighbor interaction as compared to that of the photopigment molecules alone. This may result in a sharpening of the interaction potential and hence the radial distribution function for the planar liquid-like arrangement of the antirhodopsin-photopigment complex.

Thus, our experimental results are consistent with the idea that the photopigment molecules of frog retinal receptor disk membranes most likely have a core of uniform electron density 38 to $40 \AA$ in diameter with a total diameter of 44 to $46 \AA$, and occur in a planar or two-dimensional liquid-like arrangement within the wet frog retinal receptor disk membranes.

\section{REFERENCES}

Blasie, J. K., Dewey, M. M., Blaurock, A. E. \& Worthington, C. R. (1965). J. Mol. Biol. $14,143$.

Blasie, J. K., Worthington, C. R. \& Dewey, M. M. (1969). J. Mol. Biol. 39, 407.

Dewey, M. M., Blasie, J. K., Davis, P. K. \& Barr, L. (1969). .I. Mol. Biol. 39, 407.

Guinier, A. (1963). X-ray Diffraction. London: W. H. Freeman \& Co.

Guinier, A. \& Fournet, G. (1955). Small-angle Scattering of X-rays. New York: John Wiley \& Sons, Inc.

Hill, T. L. (1956). Statistical Mechanics. New York: MeGraw-Hill Book Co., Inc.

James, R. W. (1948). The Optical Principles of the Diffraction of X-rays. Ithaca, New York: Cornell University Press.

Kaplow, R., Strong, S. L. \& Averbach, B. L. (1965). Acta Cryst. 18, 1043.

Kirkwood, J. G., Lewinson, V. A. \& Alder, B. J. (1952). J. Chem. Phys. 20, 929.

Oster, G. \& Riley, D. P. (1952). Acta Cryst. 5, 272.

Paalman, H. H. \& Pings, C. J. (1963). Rev. Mod. Phys. 35, 389.

Watson, G. N. (1944). A Treatise on the Theory of Bessel Functions, 2nd edn. New York: Cambridge University Press.

\section{APPENDICES}

The derived radial distribution functions are sensitive to two types of errors; the experimental error in the intensity measurement and the errors induced in the Fourier inversion procedures. The origin of the experimental errors have been described in the Materials and Methods section.

There are three kinds of errors induced by the Fourier inversion procedures. 
These are the intensity normalization error, the transform truncation error and the error in the scattering factor for the scattering particle concerned.

It can be shown that the upper limit of the experimental errors $( \pm 5 \%)$ carried over into the derived $2 \pi r\left\{\rho(r)-\rho_{0}\right\}_{p}$ values is $\pm 5 \%$ but the actual error is thought to be somewhat less. Hence it is permissible to analyse our derived radial distribution for nearest neighbor numbers and peak heights to at least this accuracy of $\pm 5 \%$ and considerations are given in Appendix 2 that the error concerned is somewhat less. It is also permissible to distinguish between certain model scattering factors (discussed in Appendix 4). We note that an analysis of errors induced by the Fourier inversion procedures is meaningful and the results obtained will be significant.

We first derive the radial distribution function and then discuss the formulation for the intensity normalization constant $K$, the scattering factors for the 40 to $50 \AA$ particle and the antirhodopsin molecule, and the above-mentioned errors.

\section{Appendix 1. Derivation of the Planar Radial Distribution Function}

Diffracted intensity $I\left(\mathbf{r}^{*}\right)$ arising from an electron density distribution $\sigma(\mathbf{r})$ is given by:

$$
I\left(\mathbf{r}^{*}\right)=\left|\int_{\mathrm{v}} \sigma(\mathbf{r}) \exp \left(2 \pi i \mathbf{r} \cdot \mathbf{r}^{*}\right) d \mathbf{r}\right|^{2} .
$$

Our diffraction patterns have cylindrical symmetry about an axis normal to the planes of the disk membranes and hence we resolve $\mathbf{r}$ and $\mathbf{r}^{*}$ in the usual cylindrical co-ordinates $r, \theta, Z$ and $r^{*}, \theta^{*}$ and $Z^{*}$, respectively. For our specific case, the diffracted intensity $I\left(\mathrm{r}^{*}\right)$ becomes $I\left(\mathrm{r}^{*}\right)=I\left(r^{*}\right) I\left(Z^{*}\right)$.

Suppose, for a moment, that the disk membranes consist of only a single com. ponent, the 40 to $50 \AA$ particles. Furthermore, these particles occur with a planar liquid-like arrangement and their number density may be described by a planar radial distribution function denoted by $2 \pi r \rho_{\mathrm{p}}(r)$. Then, with the beam incident normal to the planes of the disk membranes, we would record a relative intensity $I\left(r^{*}\right)$. From the diffraction theory of liquids as applied to planar liquids (see, for example, James, 1948; Oster \& Riley, 1952), equation (2) becomes:

$$
I\left(r^{*}\right)-I_{\mathrm{o}}=K_{\mathrm{p}} I_{\mathrm{p}}\left(r^{*}\right)=N f_{\mathrm{p}}^{2}\left(r^{*}\right)\left\{1-\int_{0}^{\infty} 2 \pi r\left\{\rho_{\mathrm{o}}-\rho(r)\right\}_{\mathrm{p}} J_{\mathrm{o}}\left(2 \pi r r^{*}\right) \mathrm{d} r\right\}
$$

where $I_{\mathrm{o}}$ is the unobservable diffracted intensity at the origin and $K_{\mathrm{p}} I_{\mathrm{p}}\left(r^{*}\right) \rightarrow 0$ as $r^{*} \rightarrow 0 . f_{\mathrm{p}}\left(r^{*}\right)$ is the scattering factor for each of the $N$ particles per disk membrane and $\rho_{\mathrm{o}}$ is the average density of particles in the disk membrane, i.e. $\rho_{\mathrm{o}}=N / A$ where $A$ is the area of the disk membrane. The observed relative intensity $I_{\mathrm{p}}\left(r^{*}\right)$ has been brought to an absolute scale via the intensity normalization constant $K_{\mathrm{p}}$ which is given by (see Appendix 2 for details):

$$
\frac{K_{\mathrm{p}}}{N}=\frac{\int_{0}^{\infty} 2 \pi r^{*} f_{\mathrm{p}}^{2}\left(r^{*}\right) \mathrm{d} r^{*}-\frac{N Z_{\mathrm{p}}^{2}}{A}}{\int_{0}^{\infty} 2 \pi r^{*} I_{\mathrm{p}}\left(r^{*}\right) \mathrm{d} r^{*}}
$$

where $Z_{\mathrm{p}}$ is the number of electrons per particle.

Equation (3) may be inverted by the Fourier-Bessel theorem (Watson, 1962) to give:

$$
\left\{\rho(r)-\rho_{\mathrm{o}}\right\}_{\mathrm{p}}=\int_{0}^{\infty} 2 \pi r^{*}\left\{\frac{K_{\mathrm{p}} I_{\mathrm{p}}\left(r^{*}\right)}{N f_{\mathrm{p}}^{2}\left(r^{*}\right)}-1\right\} J_{0}\left(2 \pi r r^{*}\right) \mathrm{d} r^{*} .
$$


Of course, the disk membranes do not consist of only a single component. If the 40 to $50 \AA$ particles were globular protein molecules, the other major component of the disk membrane would be phospholipid. From our birefringence data (Blasie et al., 1965; Blasie, unpublished results), the phospholipid molecules are most likely oriented with their long axis normal to the plane of the disk membrane. Hence phospholipid-phospholipid intermolecular vectors in the plane of the disk membrane would be less than $10 \AA$ in magnitude. If the 40 to $50 \AA$ particles are embedded either partially or completely in the phospholipid, the medium surrounding the particles may be considered essentially as a continuum in the treatment of low-angle X-ray diffraction data obtained with the beam incident normal to the planes of the disk membranes. If, however, the 40 to $50 \AA$ particles were spherical lipid micelles, then the other major component would most likely be amorphous non-globular protein $\dagger$ and/or Ringer's solution. Hence the medium surrounding the 40 to $50 \AA$ particles would probably consist of intermolecular vectors less than $10 \AA$ in magnitude, and again present essentially a continuum for the treatment of our low-angle diffraction data.

To see the effect of such a surrounding medium on the low-angle X-ray diffraction arising from the 40 to $50 \AA$ particles, consider the following : let the average electron density of the medium surrounding the 40 to $50 \AA$ particles be $\bar{\sigma}$; let the 40 to $50 \AA$ particles ( $p$-particles) have a cross-sectional electron density $\sigma_{\mathfrak{p}}(r)$ (i.e. $\approx$ spherical particles) with some cylindrically symmetric distribution function $\psi_{\mathrm{p}}(r)$ in the plane of the disk membrane; and let $\sigma_{\mathrm{h} . \mathrm{s}}\left(R_{\mathrm{p}}\right)$ be the cross-sectional electron density for a hard sphere of unit density and radius $R_{\mathrm{p}}$ where $R_{\mathrm{p}}$ is the maximum radius of the $p$-particles. Then for the $p$-particles embedded in the medium with mutual exclusion, we have for the electron density in the plane of the disk membrane $\sigma(r)$ where * is the convolution symbol $+\dagger$ :

$$
\sigma(r)=\bar{\sigma}-\bar{\sigma}\left\{\sigma_{\mathrm{h} . \mathrm{s} .}\left(R_{\mathrm{p}}\right) * \psi_{\mathrm{p}}(r)\right\}+\sigma_{\mathrm{p}}(r) * \psi_{\mathrm{p}}(r) .
$$

Then for the following Fourier-Bessel transforms + denoted by $\rightleftharpoons$ and spherically symmetric Fourier transforms $\ddagger \ddagger$ denoted by $\stackrel{s}{\rightleftharpoons}$ :

$$
\begin{gathered}
I\left(r^{*}\right) \rightleftharpoons \sigma(r) * \sigma(-r) \\
\bar{\sigma} \rightleftharpoons \bar{\sigma} \delta(r) \\
\sigma_{\mathrm{h} . \mathrm{s} .}\left(R_{\mathrm{p}}\right) \stackrel{\mathrm{s}}{\leftrightharpoons} f_{\mathrm{h} . \mathrm{s} .}\left(r^{*}, R_{\mathrm{p}}\right) \equiv f_{\mathrm{h} . \mathrm{s} .}\left(R_{\mathrm{p}}\right) \\
\sigma_{\mathrm{p}}(r) \stackrel{\mathrm{s}}{\leftrightharpoons} f_{\mathrm{p}}\left(r^{*}\right) \\
\psi_{\mathrm{p}}(r) \rightleftharpoons \Sigma_{\mathrm{p}}\left(r^{*}\right) .
\end{gathered}
$$

$\dagger$ We have assumed throughout these papers that the 40 to $50 \AA$ particles give rise to the two observed intensity maxima for $r^{*} \leq 0.040 \AA^{-1}$, from the wet disk membranes. This is reasonable inssmuch as upon drying of the membranes, these mexima shift in a continuous fashion in reciprocal space finally becoming the (10) and (11) reflections of a square planar lattice of 40 to $50 \AA$ particles. The existence of these particles in driod disk membranes has been independently verified by electron microscopy (Blasie et al., 1965). The absence of other intensity maxima in the low angle region suggests that there are no significant number of other globular membrane components with diameters greater than $10 \AA$.

$$
\begin{aligned}
& \dagger+a(\mathbf{r}) * b(\mathbf{r})=\int_{\mathrm{v}} a\left(\mathbf{r}^{\prime}\right) b\left(\mathbf{r}-\mathbf{r}^{\prime}\right) \mathrm{d} \mathbf{r}^{\prime} \text {. } \\
& \ddagger a(r) \leftrightharpoons A\left(r_{*}\right): a(r)=\int_{0}^{\infty} A\left(r^{*}\right) J_{0}\left(2 \pi r r^{*}\right) 2 \pi r^{*} \mathrm{~d} r^{*} \text {. } \\
& \begin{array}{c}
A\left(r^{*}\right)=\int_{0}^{\infty} a(r) J_{0}\left(2 \pi r r^{*}\right) 2 \pi r \mathrm{~d} r . \\
\vdots t(r) \leftrightharpoons T\left(r^{*}\right): t(r)=\int_{0}^{\infty} T\left(r^{*}\right) \operatorname{sinc}\left(2 \pi r r^{*}\right) 4 \pi r^{* 2} \mathrm{~d} r^{*}
\end{array} \\
& T\left(r^{*}\right)=\int_{0}^{\infty} t(r) \operatorname{sinc}\left(2 \pi r r^{*}\right) 4 \pi r^{2} \mathrm{~d} r
\end{aligned}
$$


We have for the observable diffracted intensity:

$$
I\left(r^{*}\right)-I_{\mathrm{o}}=I_{\text {obs. }}\left(r^{*}>0\right)=\left\{1-\bar{\sigma} \frac{f_{\mathrm{h} . \mathrm{s} .}\left(R_{\mathrm{p}}\right)}{f_{\mathrm{p}}\left(r^{*}\right)}\right\}^{2} f_{\mathrm{p}}^{2}\left(r^{*}\right) \Sigma_{\mathrm{p}}^{2}\left(r^{*}\right) .
$$

If the disk membrane consisted of the $p$-particles alone $(\vec{\sigma} \rightarrow 0)$, we would have in this formulation:

$$
I_{\mathrm{p}}\left(r^{*}>0\right)=f_{\mathrm{p}}^{2}\left(r^{*}\right) \Sigma_{\mathrm{p}}^{2}\left(r^{*}\right)
$$

for the observable intensity. The relationship between $I_{\mathrm{p}}\left(r^{*}\right)$ of equation (5) and $I_{\text {obs. }}\left(r^{*}\right)$ of equation (7) is given by:

$$
I_{\text {obs. }}\left(r^{*}>0\right)=\left\{1-\bar{\sigma} \frac{f_{\mathrm{h} . \mathrm{s} .}\left(R_{\mathrm{p}}\right)}{f_{\mathrm{p}}\left(r^{*}\right)}\right\}^{2} I_{\mathrm{p}}\left(r^{*}>0\right) \equiv c\left(r^{*}\right) I_{\mathrm{p}}\left(r^{*}>0\right) .
$$

For the treatment of low-angle X-ray diffraction from the $p$-particles, the presence of such a medium surrounding the $p$-particles results in a reduction of the expected diffracted intensity from the $p$-particles alone by a factor of $c\left(r^{*}\right)$.

Using equation (9), let us now write equation (5) in terms of the observed diffracted intensity, $I_{\text {obs }}\left(r^{*}>0\right)$, from the 40 to $50 \AA$ particles in the disk membrane. We then have:

$$
\begin{aligned}
& \left\{\rho(r)-\rho_{0}\right\}_{p}= \\
& \int_{0}^{\infty} 2 \pi r^{*}\left\{\left[\frac{\int_{0}^{\infty} 2 \pi r^{*} f_{\mathrm{p}}^{2}\left(r^{*}\right) \mathrm{d} r^{*}-\frac{N Z_{\mathrm{p}}^{2}}{A}}{\int_{0}^{\infty} 2 \pi r^{*} \mathrm{c}^{-1}\left(r^{*}\right) I_{\mathrm{obs} .}\left(r^{*}\right) \mathrm{d} r^{*}}\right]\left(\frac{c^{-1}\left(r^{*}\right) I_{\mathrm{obs} .}\left(r^{*}\right)}{f_{\mathrm{p}}^{2}\left(r^{*}\right)}\right)-1\right\} J_{0}\left(2 \pi r r^{*}\right) \mathrm{d} r^{*} .
\end{aligned}
$$

For any of the $\sigma_{\mathrm{p}}(r)$ models tested in Appendix 4, it may easily be shown that $f_{\mathrm{h} . \mathrm{s} .}\left(R_{\mathrm{p}}\right) / f_{\mathrm{p}}\left(r^{*}\right)$ and hence $c^{-1}\left(r^{*}\right)$ is only slowly varying with respect to $I_{\mathrm{obs} .}\left(r^{*}\right)$ in the region $r^{*} \leq r_{0}^{*}$ (we note that in the notation of Appendix 4 a $\sigma_{\mathrm{p}}(r)$ model is defined as $\sigma_{\mathrm{m}}(r)$ and then $f_{\mathrm{p}}\left(r^{*}\right)$ and $\left\{\rho(r)-\rho_{\mathrm{o}}\right\}_{\mathrm{p}}$ become $f_{\mathrm{m}}\left(r^{*}\right)$ and $\left\{\rho(r)-\rho_{\mathrm{o}}\right\}_{\mathrm{m}}$, respectively). Then, using the convolution theorem, the Fourier-Bessel transform of $\left\{c^{-1}\left(r^{*}\right)\right.$ $\left.I_{\text {obs. }}\left(r^{*}\right)\right\}$ reduces, in good approximation with less than $4 \%$ error, to just the FourierBessel transform of $I_{\text {obs. }}\left(r^{*}\right)$. Hence, we obtain:

$$
\begin{aligned}
& \left\{\rho(r)-\rho_{\mathrm{o}}\right\}_{\mathrm{p}}= \\
& \int_{0}^{\infty} 2 \pi r^{*}\left\{\left[\frac{\int_{0}^{\infty} 2 \pi r^{*} f_{\mathrm{p}}^{2}\left(r^{*}\right) \mathrm{d} r-\frac{N Z_{\mathrm{p}}^{2}}{A}}{\int_{0}^{\infty} 2 \pi r^{*} I_{\mathrm{obs} .}\left(r^{*}\right) \mathrm{d} r^{*}}\right]\left(\frac{I_{\mathrm{obs} .}\left(r^{*}\right)}{f_{\mathrm{p}}^{2}\left(r^{*}\right)}\right)-1\right\} J_{\mathrm{o}}\left(2 \pi r r^{*}\right) \mathrm{d} r^{*} \\
& =\int_{0}^{\infty} 2 \pi r^{*}\left\{\frac{K I_{\mathrm{obs} .}\left(r^{*}\right)}{N f_{\mathrm{p}}^{2}\left(r^{*}\right)}-1\right\} J_{\mathrm{o}}\left(2 \pi r r^{*}\right) \mathrm{d} r^{*}
\end{aligned}
$$

i.e. equation (1) of the section Fourier Analysis of the Diffracted Intensities where $K / N$ is defined as the term in [] in equation (11). Let us now define

$$
\left\{\frac{K I_{\text {obs. }}\left(r^{*}\right)}{N f_{\mathrm{p}}^{2}\left(r^{*}\right)}-1\right\} \equiv i_{\mathrm{p}}\left(r^{*}\right)
$$

and equation (11) becomes

$$
\left\{\rho(r)-\rho_{\mathrm{o}}\right\}_{\mathrm{p}}=\int_{0}^{\infty} 2 \pi r^{*} i_{\mathrm{p}}\left(r^{*}\right) J_{\mathrm{o}}\left(2 \pi r r^{*}\right) \mathrm{d} r^{*}
$$

or

$$
\left\{\rho(r)-\rho_{\mathrm{o}}\right\}_{\mathrm{p}} \rightleftharpoons i_{\mathrm{p}}\left(r^{*}\right) \text {. }
$$


We note that identical arguments hold for the case of the antirhodopsin serum treated disk membranes. In this case, the medium surrounding the adsorbed antirhodopsin molecules is phosphate buffered saline which is also essentially a continuum in the treatment of low-angle diffraction data. Hence, for the case of antirhodopsin serum treated disk membranes, $f_{\mathrm{p}}$ and $Z_{\mathrm{p}}$ take on their respective forms for the antirhodopsin molecule (as described in Appendix 4) in equation (12) and we then obtain $\left\{\rho(r)-\rho_{0}\right\}_{p}$ for the adsorbed antirhodopsin molecules.

\section{Appendix 2. Diffracted Intensity Normalization}

For our cylindrically symmetric system, the Patterson function $P(\mathbf{r})$ becomes $P(r) P(z)$ where $P(r)$ is given by:

$$
P(r)=\int_{0}^{\infty} 2 \pi r^{*} I\left(r^{*}\right) J_{\mathrm{o}}\left(2 \pi r r^{*}\right) \mathrm{d} r^{*}=\sigma(r) * \sigma(-r)
$$

where $\sigma(r)$ is again an electron density distribution in the plane of the disk membrane. For $r=0$, we would have:

$$
P(0)=\int_{0}^{\infty} 2 \pi r^{*} I\left(r^{*}\right) \mathrm{d} r=\int_{0}^{\infty} 2 \pi r \sigma^{2}(r) \mathrm{d} r .
$$

Again suppose that the disk membrane consists of a single component, the 40 to 50 A particles ( $p$-particles). Then:

$$
\int_{0}^{\infty} 2 \pi r \sigma^{2}(r) \mathrm{d} r=N \int_{0}^{\infty} 2 \pi r \sigma_{\mathrm{p}}^{2}(r) \mathrm{d} r
$$

But from Appendix 1, we know that:

and

$$
\sigma_{\mathrm{p}}(r)_{*} \sigma_{\mathrm{p}}(-r)=\int_{0}^{\infty} 2 \pi r^{*} f_{\mathrm{p}}^{2}\left(r^{*}\right) J_{\mathrm{o}}\left(2 \pi r r^{*}\right) \mathrm{d} r^{*}
$$

$$
\lim _{r \rightarrow 0}\left\{\sigma_{\mathrm{p}}(r)_{*} \sigma_{\mathrm{p}}(-r)\right\}=\int_{0}^{\infty} 2 \pi r \sigma_{\mathrm{p}}^{2}(r) \mathrm{d} r=\int_{0}^{\infty} 2 \pi r^{*} f_{\mathrm{p}}^{2}\left(r^{*}\right) \mathrm{d} r
$$

Also, $I\left(r^{*}\right)$ would become $I\left(r^{*}\right)=K_{\mathrm{p}} I_{\mathrm{p}}\left(r^{*}\right)+I_{\mathrm{o}}$ where $I_{\mathrm{o}}$ is again the unobserved diffraction at the origin. Then we would have:

$$
\int_{0}^{\infty} 2 \pi r^{*} K_{\mathrm{p}} I_{\mathrm{p}}\left(r^{*}\right) \mathrm{d} r^{*}+\int_{0}^{\infty} 2 \pi r^{*} I_{0} \mathrm{~d} r^{*}=N \int_{0}^{\infty} 2 \pi r^{*} f_{\mathrm{p}}^{2}\left(r^{*}\right) \mathrm{d} r^{*}
$$

Of course, $\int_{0}^{\infty} 2 \pi r^{*} I_{\mathrm{o}} \mathrm{d} r^{*}=\frac{N^{2} Z_{\mathrm{p}}^{2}}{A}$ where $Z_{\mathrm{p}}$ and $A$ have the same definitions as in Appendix 1. Hence, we have for $K_{\mathrm{p}}$ :

$$
\frac{K_{\mathrm{p}}}{N}=\frac{\int_{0}^{\infty} 2 \pi r^{*} \mathrm{f}_{\mathrm{p}}^{2}\left(r^{*}\right) \mathrm{d} r^{*}-\frac{N Z_{\mathrm{p}}^{2}}{A}}{\int_{0}^{\infty} 2 \pi r^{*} I_{\mathrm{p}}\left(r^{*}\right) \mathrm{d} r^{*}}
$$

Then, using equation (9) and the arguments presented in Appendix 1 where $c^{-1}\left(r^{*}\right)$ is slowly varying, we have:

$$
\frac{K}{N}=\frac{\int_{0}^{\infty} 2 \pi r^{*} f_{\mathrm{p}}^{2}\left(r^{*}\right) \mathrm{d} r^{*}-\frac{N Z_{\mathrm{p}}^{2}}{A}}{\int_{0}^{\infty} 2 \pi r^{*} I_{\mathrm{obs} .}\left(r^{*}\right) \mathrm{d} r^{*}}
$$

for the case of the 40 to $50 \AA$ particles occurring in a natural disk membrane. The factor $N / A$ may be replaced by $A_{0}$ where $A_{\mathrm{o}}$ is the average area of the disk membrane available to one particle. $A_{0}$ may be easily determined assuming that the total 
number of particles per disk membrane is conserved upon partial drying and we know that in the partially dried state one particle occurs per unit cell, i.e. per each $4900 \AA^{2}$.

Thus, with the particle scattering factors from Appendix 4, the diffracted intensities may be normalized for the 40 to $50 \AA$ particles of the receptor disk membrane.

In the previous paper (Blasie et al., 1969), we show that the diffracted intensity from the antirhodopsin serum treated disk membranes may be considered to arise solely from the antirhodopsin molecules adsorbed to the disk membranes. Hence, using the scattering factor for the cylindrically symmetric antirhodopsin molecule $\left[f_{\mathrm{p}}\left(\mathrm{r}^{*}\right)\right]$ cylindrically symmetric about the $Z^{*}$ axis also reduces to $f_{\mathrm{p}}\left(r^{*}\right)$ in the $Z^{*}=0$ projection) and using a procedure identical to that outlined above, the diffracted intensities from the serum treated disk membranes may also be normalized.

The integrals occurring in equation (17) are also terminated at $r_{0}^{*}$. This truncation error as well as errors associated with particle scattering factors and observed intensity measurement may well induce errors in the intensity normalization constant.

For the treatment of intensity normalization errors in spherically symmetric systems, see, for example, the presentation of Paalman \& Pings (1963). In our planar system, for an error in intensity normalization of $\epsilon$ such that the normalization constant $K$ becomes $K(1+\epsilon)$ and using Fourier transforms cylindrically symmetric about the $Z^{*}$ axis, one can easily show that the calculated radial distribution function $2 \pi r \rho_{\text {calc }}(r)$ containing the normalization constant $K(1+\epsilon)$ is related to the true radial distribution function $2 \pi r \rho_{\text {true }}(r)$ containing the normalization constant $K$ as follows:

$$
2 \pi r\left\{\rho_{\mathrm{calc}}(r)-\rho_{0}\right\}_{\mathrm{p}}=2 \pi r(1+\epsilon)\left\{\rho_{\mathrm{true}}(r)-\rho_{\mathrm{o}}\right\}_{\mathrm{p}}+2 \pi \epsilon r_{\mathrm{o}}{ }^{*} J_{1}\left(2 \pi r r_{\mathrm{o}}{ }^{*}\right) .
$$

As an independent check on the intensity normalization constant, one can show that $K I_{\text {obs. }}\left(r^{*}\right) \rightarrow N f_{\mathrm{p}}^{2}\left(r^{*}\right)$ for $r^{*} \geqslant r_{\mathrm{o}}{ }^{*}$. For $r^{*}>r_{\mathrm{o}}{ }^{*}=0.024 \AA^{-1}$, the $K / N$ derived in this Appendix and $f_{\mathrm{p}}\left(r^{*}\right)$ calculated in Appendix 4 resulted in:

$$
\frac{\mid \frac{K}{N} I_{\text {obs. } .\left(r^{*}\right)-f_{\mathrm{p}}^{2}\left(r^{*}\right) \mid}}{f_{\mathrm{p}}^{2}\left(r^{*}\right)}<0.02 \text { for } r^{*} \geqslant 0.024 \AA^{-1}
$$

and hence $\epsilon<0.02$. With $r_{0}^{*}=0.024 \AA^{-1}$, the major contribution from the second term in equation (11) is confined to the region where, $r \leq 25.0 \AA$. However, these contributions are small $(\epsilon<0.02)$ and the small oscillations for small $r$ in the radial distribution function $2 \pi r \rho_{\mathrm{p}}(r)$ as calculated from equation (1) are not characteristic of $J_{1}(x)$ about the origin and can be completely accounted for by transform truncation errors and particle scattering factor errors (see following Appendices). Thus, apparently only the first term, the linear perturbation of $\left\{2 \pi r \rho_{\text {true }}(r)\right\}_{\mathrm{p}}$ on the right side of equation (18) limits our determination of nearest neighbor peak heights and nearest neighbor numbers to within $\pm 2 \%$.

\section{Appendix 3. Transform Truncation}

With reference to equation (12), of Appendix 1, we see that $\rho_{\mathrm{p}}(r)$ of the true radial distribution function is given by the following equation:

$$
\left\{\rho(r)-\rho_{0}\right\}_{\mathrm{p}}=\int_{0}^{\infty} 2 \pi r^{*} i_{\mathrm{p}}\left(r^{*}\right) J_{0}\left(2 \pi r r^{*}\right) \mathrm{d} r^{*}
$$


In practice, the integral is terminated at some $r_{\mathrm{o}}^{*}$ where $K I_{\mathrm{obs}}\left(r^{*}\right) \rightarrow N f_{\mathrm{p}}^{2}\left(r^{*}\right)$ i.e. $i_{p}\left(r^{*}\right) \rightarrow 0$ for $r^{*}>r_{0}^{*}$ which results in the presence of a Fourier-Bessel component of minimum wavelength, in the truncated radial distribution function denoted in primed notation as follows:

$$
\left\{\rho^{\prime}(r)-\rho_{0}\right\}_{\mathrm{p}}=\int_{0}^{\mathrm{r}_{0}^{*}} 2 \pi r^{*} i_{\mathrm{p}}\left(r^{*}\right) J_{0}\left(2 \pi r r^{*}\right) \mathrm{d} r^{*}
$$

Equation (20) is equivalent to the following:

$$
\left\{\rho^{\prime}(r)-\rho_{0}\right\}_{\mathrm{p}}=\int_{0}^{\infty} 2 \pi r^{*} w\left(r^{*}\right) i_{\mathrm{p}}\left(r^{*}\right) J_{0}\left(2 \pi r r^{*}\right) \mathrm{d} r^{*}
$$

where

$$
w\left(r^{*}\right)=\left\{\begin{array}{l}
1 \text { for } r^{*} \leq r_{0}^{*} \\
0 \text { for } r^{*}>r_{0}^{*}
\end{array}\right.
$$

The Fourier-Bessel transforms (denoted by $\rightleftharpoons$ ) of $i_{\mathrm{p}}\left(r^{*}\right)$ and $w\left(r^{*}\right)$, are:

$$
\begin{gathered}
i_{\mathrm{p}}\left(r^{*}\right) \rightleftharpoons\left\{\rho(r)-\rho_{0}\right\}_{\mathrm{p}} \\
w\left(r^{*}\right) \rightleftharpoons 2 \pi\left(r_{\mathrm{o}}^{*}\right)^{2} \frac{J_{1}\left(2 \pi r r_{0}^{*}\right)}{2 \pi r r_{0}^{*}} \equiv W(r) .
\end{gathered}
$$

The convolution theorem allows us to write equation (21) as:

$$
\begin{aligned}
&\left\{\rho^{\prime}(r)-\rho_{0}\right\}_{\mathrm{p}}=\int_{0}^{+\infty}\left\{\rho\left(r^{\prime}\right)-\rho_{0}\right\}_{\mathrm{p}} 2 \pi\left(r_{\mathrm{o}}^{*}\right)^{2} \frac{J_{1}\left\{2 \pi\left(r-r^{\prime}\right) r_{\mathrm{o}}^{*}\right\}}{\left\{2 \pi\left(r-r^{\prime}\right) r_{\mathrm{o}}^{*}\right.} 2 \pi r^{\prime} \mathrm{d} r^{\prime} \\
&=\int_{0}^{+\infty}\left\{\rho\left(r^{\prime}\right)-\rho_{0}\right\}_{\mathrm{p}} W\left(r-r^{\prime}\right) 2 \pi r^{\prime} \mathrm{d} r^{\prime}=\left\{\rho(r)-\rho_{0}\right\}_{\mathrm{p}} * W(r) .
\end{aligned}
$$

To see the effect of transform truncation on $2 \pi r \rho_{p}(r)$, we suppose for the moment that $\rho_{\mathrm{p}}(r)$ may be approximated by a sharply varying (with respect to $W(r)$ ) maxima in the neighborhood of the first nearest neighbor peak at $r_{1}$ and the area under this maxima is $N_{1}$. Then, from convolution theory, equation (23) becomes approximately [with less than $10 \%$ error if the half-width of $\rho_{\mathcal{p}}(r)$ is less than three-tenths the half-width of $W(r)]$ :

$$
\rho_{\mathrm{p}}^{\prime}(r) \approx N_{1} W\left(r-r_{1}\right)=N_{1} 2 \pi\left(r_{0}^{*}\right)^{2} \frac{J_{1}\left\{2 \pi\left(r-r_{1}\right) r_{\mathrm{n}}^{*}\right\}}{\left\{2 \pi\left(r-r_{1}\right) r_{\mathrm{o}}^{*}\right\}}
$$

and:

$$
2 \pi r \rho_{\mathrm{p}}^{\prime}(r) \approx 2 \pi r N_{1} W\left(r-r_{1}\right) .
$$

For $r_{\mathrm{o}}{ }^{*}=0.024 \AA^{-1}$, Figure 5 shows the effect of transform truncation on the true radial distribution function about its first nearest neighbor maxima at $r_{1}$. Thus the truncated radial distribution function exhibits a first nearest neighbor maxima which may be broader and of lesser amplitude than that of the true radial distribution function. However, we have shown (Blasie \& Worthington, 1968, Abstr. Ann. Meeting Biophys. Soc.) that model radial distribution functions, in which nearest neighbor numbers were conserved with respect to the radial distribution functions derived from the diffracted intensities as the nearest neighbor peak half-widths were varied, which predicted the normalized observed intensities with reliability factors of less than $10 \%$ had nearest neighbor peak half-widths only slightly smaller than those of the distribution functions determined from the diffracted intensities. Such reliability factors were calculated in the usual sense sampling the experimental and predicted intensity functions at identical intervals of $\Delta r^{*}=0.001 \AA^{-1}$. Thus, the 


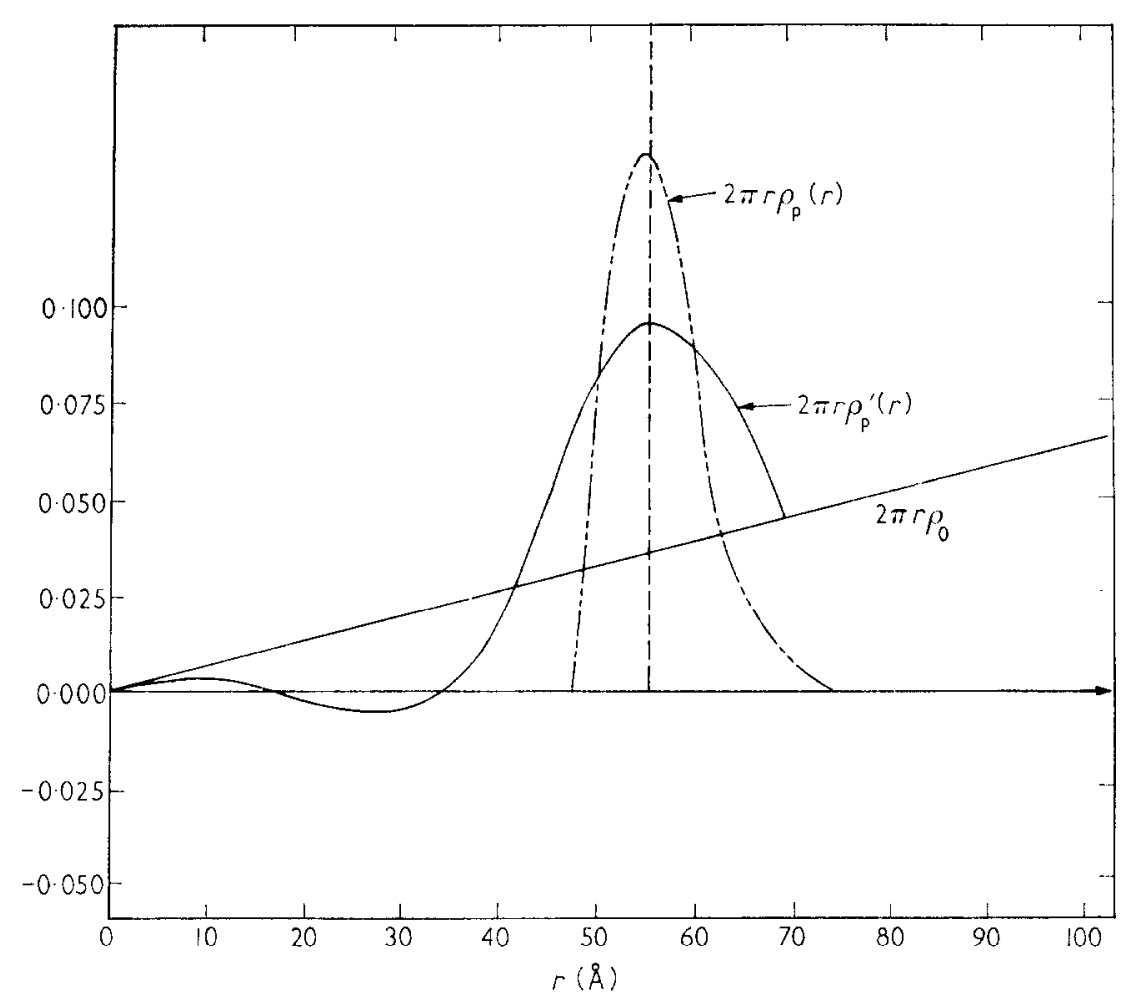

Fig. 5. Effects of transform truncation about the first nearest neighbor peak in the region of the planar radial distribution function where $r$ is less than the particle diameter. Truncation effects were calculated according to Appendix 3, for comparison with the planar radial distribution function of 40 to $50 \mathrm{~A}$ particles of the untreated disk membranes at $26.0^{\circ} \mathrm{C}$.

primary effect of transform truncation as seen in Figure 5 is to induce small amplitude oscillations of the approximate form,

$$
r \frac{J_{1}\left\{2 \pi\left(r-r_{1}\right) r_{o}^{*}\right\}}{\left\{2 \pi\left(r-r_{1}\right) r_{\mathrm{o}}^{*}\right\}}
$$

about the first nearest neighbor peak at $r_{1}$ in the derived radial distribution functions in the region for $r$ less than the particle diameter where the function should be zero. It is apparent from equations (23), (24) and (25) that the effect of transform truncation on only the first nearest neighbor peak results in significant contribution in the small- $r$ region and hence Figure 5 shows the probable form of such oscillations for small $r$.

It will be shown in the following Appendix that errors in the particle scattering factor also affect the radial distribution function primarily in the region of $r$ less than the particle diameter. A method will be prescribed for the elimination of these errors thereby obtaining the "true" scattering factor for the scattering particle concerned. It is apparent that after the elimination of the particle scattering factor errors, one will still be left with the transform truncation error in the region of small $r$.

It should also be noted that according to Appendix $1, K_{\mathrm{p}} I_{\mathrm{p}}\left(r^{*}\right) \rightarrow 0$ as $r^{*} \rightarrow 0$ and hence, so must $K I_{\text {obs. }}\left(r^{*}\right)$ according to the arguments presented there. Of course, 
we do not record $I_{\text {obs. }}\left(r^{*}\right)$ near the origin and hence $I_{\text {obs. }}\left(r^{*}\right)$ must be extrapolated to zero as $r^{*} \rightarrow 0$. It may easily be shown that an error in this extrapolation will add a low frequency oscillation (of period 140 to $150 \AA$ ) of extremely small amplitude in the derived radial distribution function. For our purposes, its effect on the derived radial distribution function is negligible.

\section{Appendix 4. Particle Scattering Factors}

For the treatment of particle scattering factor errors in the spherically symmetric case, see, for example, Kaplow, Strong \& Averbach, 1965. For our cylindrically symmetric system, we suppose that the true particle scattering factor $f_{\mathrm{p}}\left(r^{*}\right)$ is related to the model particle scattering factor $f_{\mathrm{m}}\left(r^{*}\right)$ through a slowly varying error function $\epsilon\left(r^{*}\right)$ as follows:

$$
f_{\mathrm{p}}^{2}\left(r^{*}\right)=\left\{1+\epsilon\left(r^{*}\right)\right\} f_{\mathrm{m}}^{2}\left(r^{*}\right) .
$$

Then, from equation (21) of Appendix 3 we have:

$$
\rho_{\mathrm{p}, \mathrm{m}}^{\prime}(r)-\rho_{0}=\int_{0}^{\infty} 2 \pi r^{*} w\left(r^{*}\right) i_{\mathrm{p}, \mathrm{m}}\left(r^{*}\right) J_{0}\left(2 \pi r r^{*}\right) \mathrm{d} r .
$$

Substitution of equation (26) leads to:

$$
\begin{aligned}
\rho_{\mathrm{m}}^{\prime}(r)-\rho_{0} & =\int_{0}^{\infty} 2 \pi r^{*}\left\{1+\epsilon\left(r^{*}\right)\right\} w\left(r^{*}\right) i_{\mathrm{p}}\left(r^{*}\right) J_{0}\left(2 \pi r r^{*}\right) \mathrm{d} r \\
& +\int_{0}^{\infty} 2 \pi r^{*} w\left(r^{*}\right) \epsilon\left(r^{*}\right) J_{0}\left(2 \pi r^{*}\right) \mathrm{d} r^{*}
\end{aligned}
$$

For :

$$
\begin{aligned}
& i_{\mathrm{p} . \mathrm{m}}\left(r^{*}\right) \rightleftharpoons\left\{\rho_{\mathrm{p} . \mathrm{m}}(r)-\rho_{0}\right\}, w\left(r^{*}\right) \rightleftharpoons W(r), \text { and defining } \\
& \left\{1+\epsilon\left(r^{*}\right)\right\} \rightleftharpoons H(r), \text { and } \epsilon\left(r^{*}\right) \rightleftharpoons E(r),
\end{aligned}
$$

we have:

$$
\left\{\rho_{m}^{\prime}(r)-\rho_{0}\right\}=H(r)_{*} W(r)_{*}\left\{\rho_{\mathrm{p}}(r)-\rho_{0}\right\}+W(r)_{*} E(r) .
$$

Remembering that $W(r) *\left\{\rho_{\mathrm{p}}(r)-\rho_{0}\right\}=\left\{\rho_{\mathrm{p}}^{\prime}(r)-\rho_{0}\right\}$ and for $\epsilon\left(r^{*}\right)$ slowly varying, $H(r) *\left\{\rho_{\mathrm{p}}^{\prime}(r)-\rho_{0}\right\} \approx\left\{\rho_{\mathrm{p}}^{\prime}(r)-\rho_{0}\right\}$. Thus equation (29) becomes:

$$
\begin{gathered}
\left\{\rho_{\mathrm{m}}^{\prime}(r)-\rho_{\mathrm{p}}^{\prime}(r)\right\} \approx W(r)_{*} E(r) \\
\approx \int_{0}^{\infty} 2 \pi r^{*} w\left(r^{*}\right) \epsilon\left(r^{*}\right) J_{0}\left(2 \pi r^{*}\right) \mathrm{d} r \approx \int_{0}^{\mathrm{r}_{0}^{*}} 2 \pi r^{*} \epsilon\left(r^{*}\right) J_{0}\left(2 \pi r r^{*}\right) \mathrm{d} r^{*} .
\end{gathered}
$$

It is apparent that for $r_{0}^{*}=0.024 \AA^{-1}$, the major contribution to the right side of equation (22) occurs for $r \leq 25 \AA$ due to the nature of $J_{0}(x)$. For the 40 to $50 \AA$ particles of the receptor disk membranes and the $32 \AA$ diameter antirhodopsin molecules adsorbed to the antirhodopsin serum treated disk membranes, this contribution occurs well within the particle diameter. Thus errors in the particle scattering factor will induce an oscillatory behavior in $2 \pi r \rho_{p}(r)$ of equation (1) in the section Fourier Analysis of the Diffracted Intensities, for small $r$ in addition to those of transform truncation through the convolution of the two errors. In absence of particle scattering factor errors $\left[\epsilon\left(r^{*}\right)=0\right.$ for all $r^{*}$ and hence $E(r)=0$ for all $\left.r\right]$ reduces to $W(r)_{*} E(r)=0$ and then $\rho_{m}^{\prime}(r)=\rho_{\mathrm{p}}^{\prime}(r)$. Hence, the best possible particle scattering factor obtained by parameter variation of a model particle scattering factor will be the one which best results in a behavior of $2 \pi r \rho_{\mathrm{m}}^{\prime}(r)$ for small $r$ as that predicted by transform truncation alone, namely $2 \pi r \rho_{p}^{\prime}(r)$ of equation (25) Appendix 3. 
Model particle scattering factors for a 40 to $50 \AA$ globular protein molecule were based on the following cross-sectional electron densities $\sigma(r)$ for the molecule:

(i) hard sphere $\sigma_{\mathrm{m}}(r)=\left\{\begin{array}{l}1 \text { for } r \leq R_{\mathrm{m}} \text { (hard core molecular diameter) } \\ 0 \text { for } r>R_{\mathrm{m}}\end{array}\right.$

(ii) hard sphere + exponential decay

$$
\sigma_{\mathrm{m}}(r)=\left\{\begin{array}{l}
1 \text { for } r \leq R_{\mathrm{m}} \\
\mathrm{e}^{-\alpha\left(r-R_{\mathrm{m}}\right)} \text { for } r>R_{\mathrm{m}}
\end{array}\right.
$$

(iii) soft sphere $\sigma_{\mathrm{m}}(r)=\left\{\mathrm{e}^{a\left(\mathrm{r}-{ }^{R} \mathrm{~m}\right)}+1\right\}^{-1}$

(for ii and iii, $\alpha$ is a constant of dimension $\AA^{-1}$ ).

Considering the 40 to $50 \AA$ particle as a spherical lipid micelle, a cross-sectional electron density $\sigma_{m}(r)$ for the spherical micelle of $\sigma_{m}(r)=e^{-a r}$ seemed appropriate.

Spherically symmetric Fourier transforms of the various cross-sectional electron density models for the 40 to $50 \AA$ particles, i.e. the particle scattering factors, were calculated and were then normalized to the molecular (or "micellar") number $Z_{\mathrm{m}}$

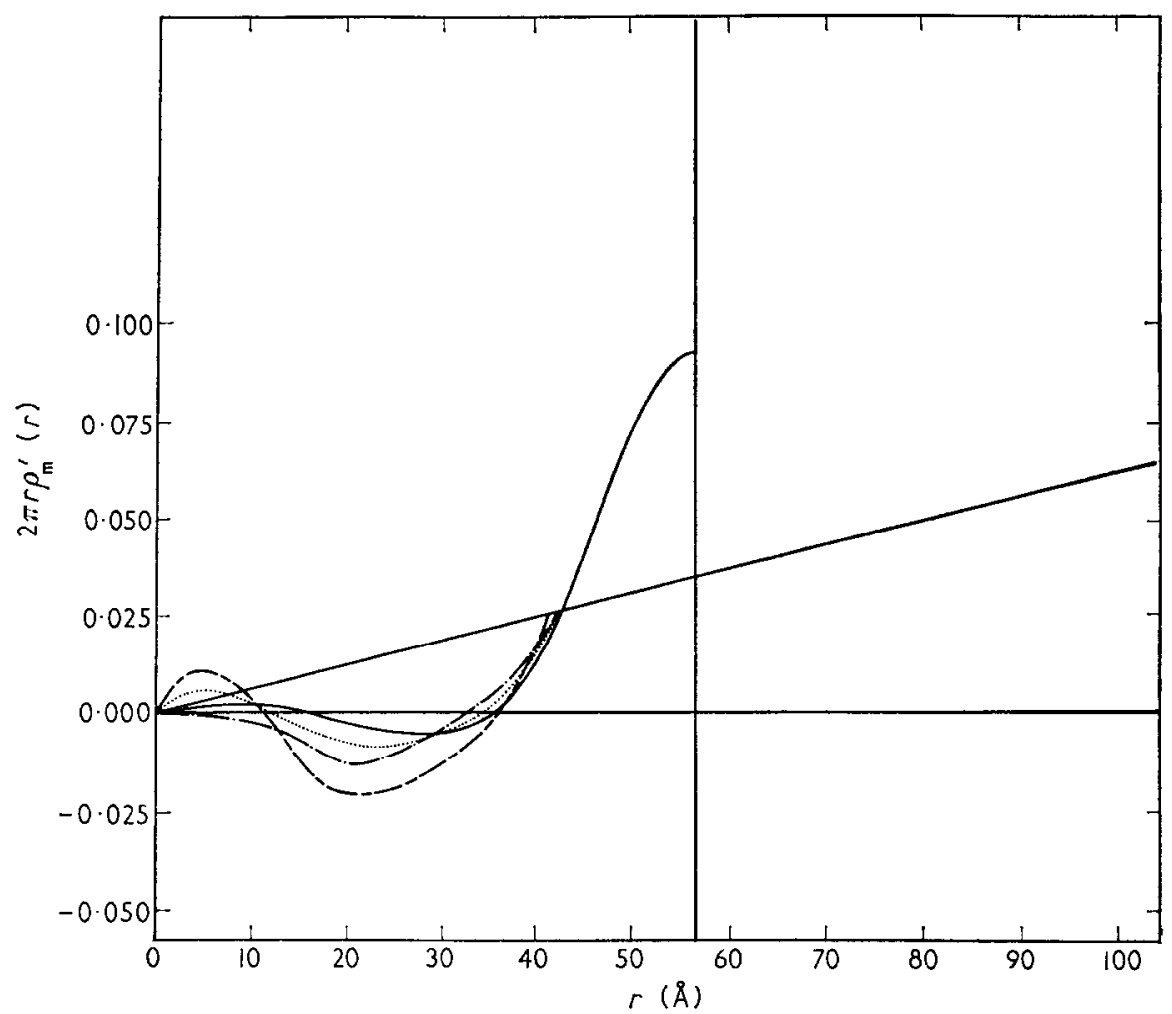

FrG. 6. Planar radial distribution function for the particles of the wet untreated disk membranes in the region where $r$ is less than the particle diameter. Best model particle scattering factors based on hard-sphere and soft-sphere cross-sectional electron density models for the particle were used. These planar functions are compared with that predicted by transform truncation alone about the first nearest neighbor peak in the region where $r$ is less than the particle diameter. --- , Hard sphere model $R_{m}=22 \AA ;-.-$, hard sphere model $R_{\mathrm{m}}=20 \AA ; \ldots$, soft. sphere model $R_{\mathrm{m}}=21 \AA, \alpha=10 \AA ; \perp$, transform truncation alone. 
at the origin. For the globular protein model scattering factors, the molecular number was calculated using a density of 1.3 to $1.4 \mathrm{~g} / \mathrm{cc}$, a molecular volume of $\frac{4}{3} \pi R_{\mathrm{m}}^{3}$, and $Z_{m}$ was then approximately one-half the molecular weight corrected for $7 \%$ hydrogen. For the spherical lipid micelle of density $0.9 \mathrm{~g} / \mathrm{cc}$, "micellar numbers" were calculated using a micellar volume of $\frac{4}{3} \pi R_{\mathrm{m}}^{3}$ (where $R_{\mathrm{m}}$ was defined in this case as that $r$ such that $\sigma(r)<0.1$ for $r>R_{\mathrm{m}}$ ), and $Z_{\mathrm{m}}$ was approximately one-half the "micellar weight" corrected for $10 \%$ hydrogen. With these model scattering factors, the radial distribution functions were calculated from the normalized diffracted intensities at $26 \cdot 0^{\circ} \mathrm{C}$ with variation of $R_{\mathrm{m}}\left(5 \AA \leq R_{\mathrm{m}} \leq 60 \AA\right)$ and $\alpha\left(0 \cdot 1 \AA^{-1} \leq \alpha \leq 10 \cdot 0 \AA^{-1}\right)$ and were subsequently examined in the region of $r<2 R_{m}$. It was found that only the particle scattering factors based on $\sigma_{\mathrm{m}}(r)$ of the hard-sphere models (20 $\AA \leq R_{\mathrm{m}} \leq$ $22 \AA$ ) and soft-sphere models with $20 \AA \leq R_{\mathrm{m}} \leq 21 \AA$ and $\alpha \geq 5 \AA^{-1}$ gave radial distribution functions $2 \pi r \rho_{m}^{\prime}(r)$ which approached that predicted from transform truncation alone for small $r$. Thus, only reasonably hard soft-sphere models gave the best scattering factors of the soft-sphere models and the calculated radial distribution function $2 \pi r \rho_{m}^{\prime}(r)$ for that $\sigma_{m}(r)$ model with $R_{m}=21 \AA$ and $\alpha=10 \AA^{-1}$ is compared with that predicted by transform truncation alone in Figure 6. Slight differences between the two distribution functions are evident for $r<20 \AA$. This difference will be nearly removed using another method to be discussed later. At this point, it is apparent that a reasonably hard soft-sphere based model scattering factor for a globular protein molecule with a hard core of uniform electron density 38 to $40 \AA$

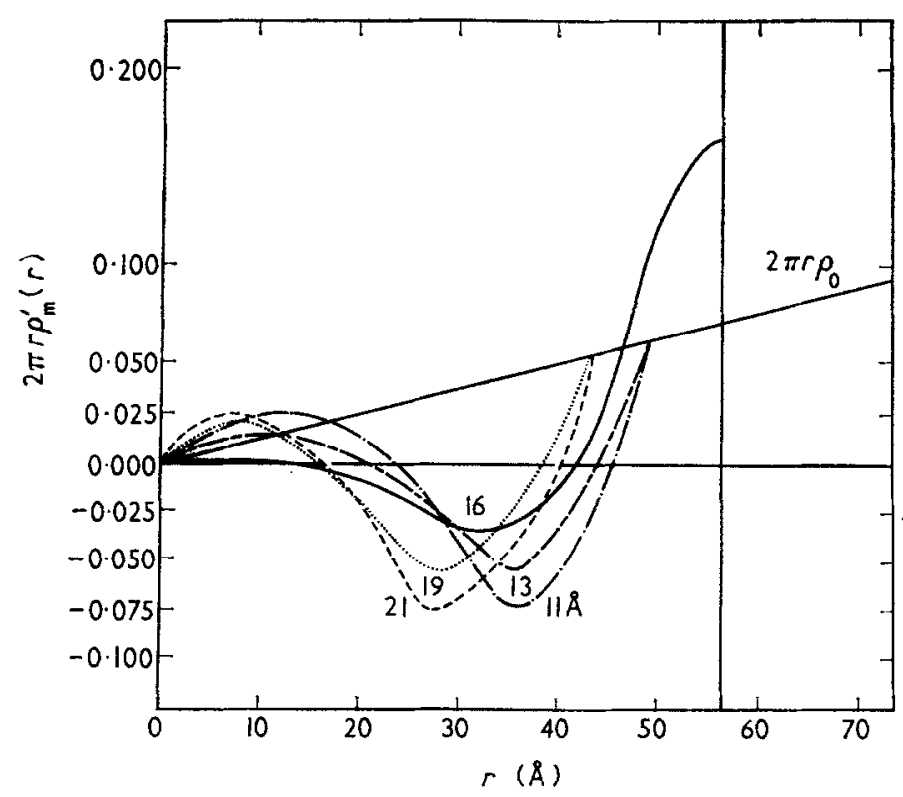

FrG. 7. Planar radial distribution functions for the cylindrical antirhodopein moleoules adsorbed to the wet antirhodopsin serum treated disk membranes using partiale ecattering factors based on hard oylinder cross-eectional electron density models for the antirhodopsin molecules. The effect of variation of the oylinder radius on the planar function is shown in the region for $r$ less than the molecular diemeter.

Hard-cylinder models: $-\left[, R_{\mathrm{m}}=16 \AA ;-., R_{\mathrm{m}}=11 \AA ;---, R_{\mathrm{m}}=13 \AA ; \ldots R_{\mathrm{m}}\right.$ $=19 A_{;}----, R_{\mathrm{m}}=21 \mathrm{~A}$. 


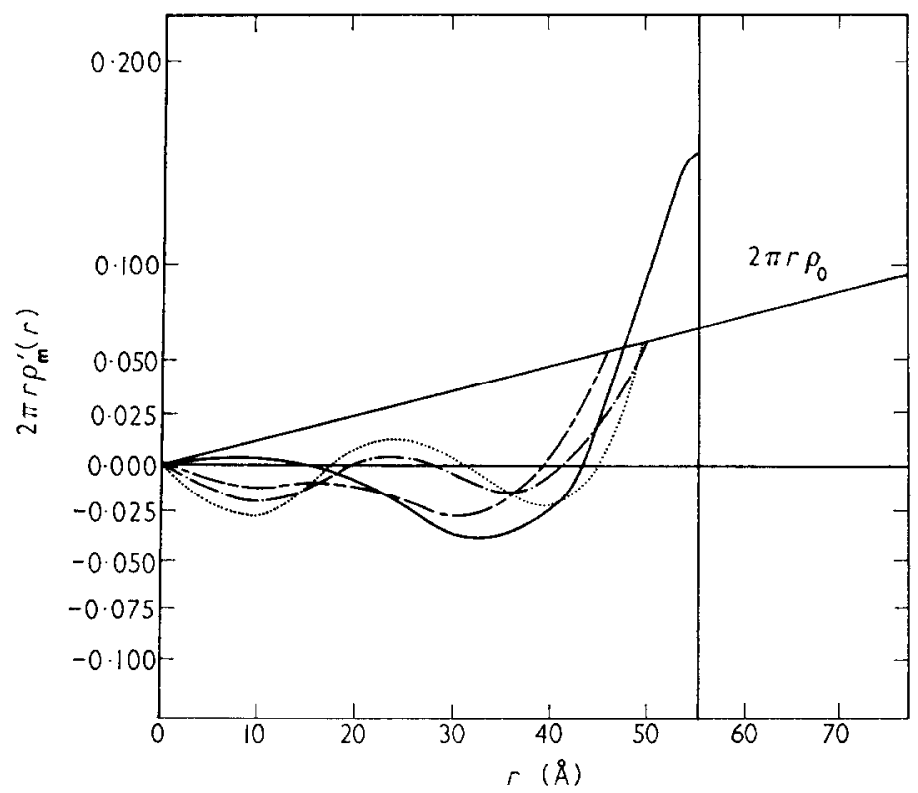

Fig. 8. Same as Fig. 7 except that the particle scattering factors were based on soft-cylinder cross-sectional electron density models for the antirhodopsin molecules. The effects of variation of the effective cylinder radius on the planar radial distribution function are shown for $r$ less than the molecular diameter. Variation of the degree of "softness" demonstrated that only reasonably hard $\left(\alpha \geqslant 5 \AA^{-1}\right.$ ) soft-cylinder models approached the proper behavior for the small r-region.

..... Soft-cylinder model $R_{\mathrm{m}}=14 \AA, \alpha=10 \AA^{-1} ;-\cdot-$, soft-cylinder model $R_{\mathrm{m}}=16 \AA$,

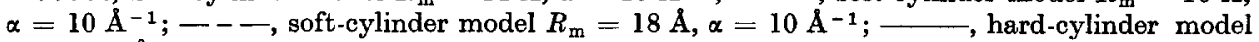
$R_{\mathrm{m}}=16 \AA$.

in diameter with a total diameter of 44 to $46 \AA$ is quite good while others, particularly that for a spherical lipid micelle, result in severe oscillations in $2 \pi r \rho_{m}^{\prime}(r)$ for $r<2 R_{m}$. The transform truncation error was identical in all cases.

For the antirhodopsin molecule adsorbed to the antirhodopsin serum treated disk membrane, it was found in an entirely analogous fashion that a molecular scattering factor obtained by a Fourier transform cylindrically symmetric about the axis of a hard cylinder electron density model with a radius $R_{\mathrm{m}}=16 \cdot 0 \pm 1 \AA$ and a length $l=240 \pm 10 \AA$ (molecular volume of $\pi R^{2}{ }_{\mathrm{m}} l$, density of 1.3 to $1.4 \mathrm{~g} / \mathrm{cc}$, and $Z_{\mathrm{m}}$ approximately one-half the molecular weight corrected for $7 \%$ hydrogen) gave a radial distribution function $2 \pi r \rho_{\mathrm{m}}^{\prime}(r)$ which best approached that predicted from transform truncation alone see (Figs. 7 and 8).

The molecular scattering factors as derived above were used in calculating the radial distribution functions shown in Figures 3 and 4.

Another approach to the calculation of the true particle scattering factor is via a Fourier-Bessel inversion of equation (30). First, we observe that our best $f_{\mathrm{m}}\left(r^{*}\right)$ based on a soft-sphere $\sigma(r)$ model with $R_{\mathrm{m}}=21 \cdot 0 \AA$ and $\alpha=10 \cdot 0 \AA^{-1}$ resulted in $2 \pi r \rho_{\mathrm{m}}^{\prime}(r)$-such that $2 \pi r \rho_{\mathrm{p}}^{\prime}(r)$ was still fairly small compared with $2 \pi r \rho_{\mathrm{m}}^{\prime}(r)$ in the small $r$ region. Hence, using $f_{\mathrm{m}}\left(r^{*}\right)$ based on soft-sphere $\sigma_{\mathrm{m}}(r)$ models, we can assume the left side of equation (24) to be approximately $\rho_{m}^{\prime}(r)$. Then, a Fourier-Bessel inversion of equation (30) in the small $r$ region gives:

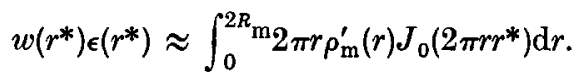


For $r^{*} \leq r_{0}^{*}\left(w\left(r^{*}\right) \equiv 1\right.$ for $\left.r^{*} \leq r_{0}^{*}\right)$, then

$$
\epsilon\left(r^{*}\right) \approx \int_{0}^{2 R \mathrm{~m}} 2 \pi r \rho_{\mathrm{m}}^{\prime}(r) J_{0}\left(2 \pi r r^{*}\right) \mathrm{d} r
$$

and for $r_{0}^{*}>r_{0}^{*}, \epsilon\left(r^{*}\right)$ is undetermined.

With the various approximations leading to equation (32), we do not calculate a precise $\epsilon\left(r^{*}\right)$. Instead we obtain an approximation to it, namely $\epsilon_{\mathfrak{p} 1}\left(r^{*}\right)$. In this manner, $\epsilon_{\mathrm{p} 1}\left(r^{*}\right)$ was obtained from several $\rho_{\mathrm{m}}^{\prime}(r)$ based on the soft-sphere molecular scattering factor $\left(\alpha=10 \AA^{-1}\right.$ ) for the 40 to $50 \AA$ globular protein molecule varying $R_{\mathrm{m}}\left(10 \AA \leq R_{\mathrm{m}} \leq 30 \AA\right)$, then $f_{\mathrm{p} 1}^{2}\left(r^{*}\right)$ was calculated via equation (26) and then $2 \pi r \rho_{p 1}^{\prime}(r)$ was calculated via equation (27). It was found that the $\epsilon_{p 1}\left(r^{*}\right)$ derived from $\rho_{\mathrm{m}}^{\prime}(r)$ based on a soft-sphere model molecular scattering factor $f_{\mathrm{m}}\left(r^{*}\right)$ with $R_{\mathrm{m}}=21 \cdot 0 \AA\left(\alpha=10 \AA^{-1}\right)$ induced the most rapid convergence to a $f_{\mathrm{p} 1}^{2}\left(r^{*}\right)$ such that $2 \pi r \rho^{\prime}{ }_{p 1}(r)$ became nearly identical to that predicted by transform truncation alone, $2 \pi r \rho_{\mathrm{p}}^{\prime}(r) . f_{\mathrm{p} 1}^{2}\left(r^{*}\right)$ and $f_{\mathrm{m}}^{2}\left(r^{*}\right)$ are shown in Figure 8 and $2 \pi r \rho_{\mathrm{p} 1}^{\prime}(r)$ together with

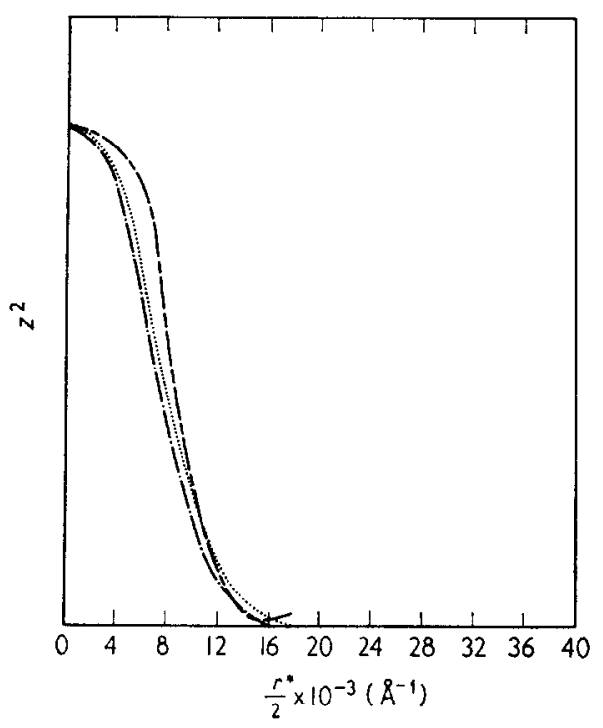

FIG. 9. The shape of the square of the "true" particle scattering factor $\left\{f_{\mathrm{pl}}^{2}\left(r^{*}\right)\right\}$ for the particles of the wet untreated disk membranes is compared here with that of the square of the best model particle scattering factor $\left\{f_{\mathrm{m}}^{2}\left(r^{*}\right)\right\}$ based on a soft-sphere cross-sectional electron density for the perticles. Both $f_{\mathrm{pl}}^{2}$ and $f_{\mathrm{m}}^{2}$ were determined by the methods described in Appendix 4.

- - , Hard-sphere model $f_{\mathrm{m}}^{2}\left(r^{*}\right) R_{\mathrm{m}}=22 \AA ; \ldots$, soft-sphere model $f_{\mathrm{m}}^{2}\left(r^{*}\right) R_{\mathrm{m}}=21 \AA$, $\alpha=10 \AA^{-1} ;----, f_{\mathrm{pl}}^{2}\left(r^{*}\right)$.

the $2 \pi r \rho_{p}^{\prime}(r)$ predicted from transform truncation alone are shown in Figure 9. However, as $\rho_{\mathrm{m}}^{\prime}(r)$ is limited in resolution by $r_{0}^{*}$, so is the true molecular scattering factor known accurately only for $r^{*}<r_{\mathrm{o}}{ }^{*}$. Thus, inversion of $f_{\mathrm{p} 1}\left(r^{*}\right)$ to obtain the true cross-sectional electron density for the globular protein molecule is not too meaningful. Therefore, the shape of the true scattering factor for $r^{*} \leq r_{0}^{*}$ for the globular protein which we have shown to be the photopigment molecule is shown in Figure 9 while the cross-sectional electron density for the photopigment molecule 
is well described by a soft-sphere model where the hard core of uniform electron density is about 38 to $40 \AA$ in diameter and the total diameter is 44 to $46 \AA$. By comparison with our electron microseopy, it is apparent that the hard core of uniform electron density is the non-polar core of the photopigment molecule.

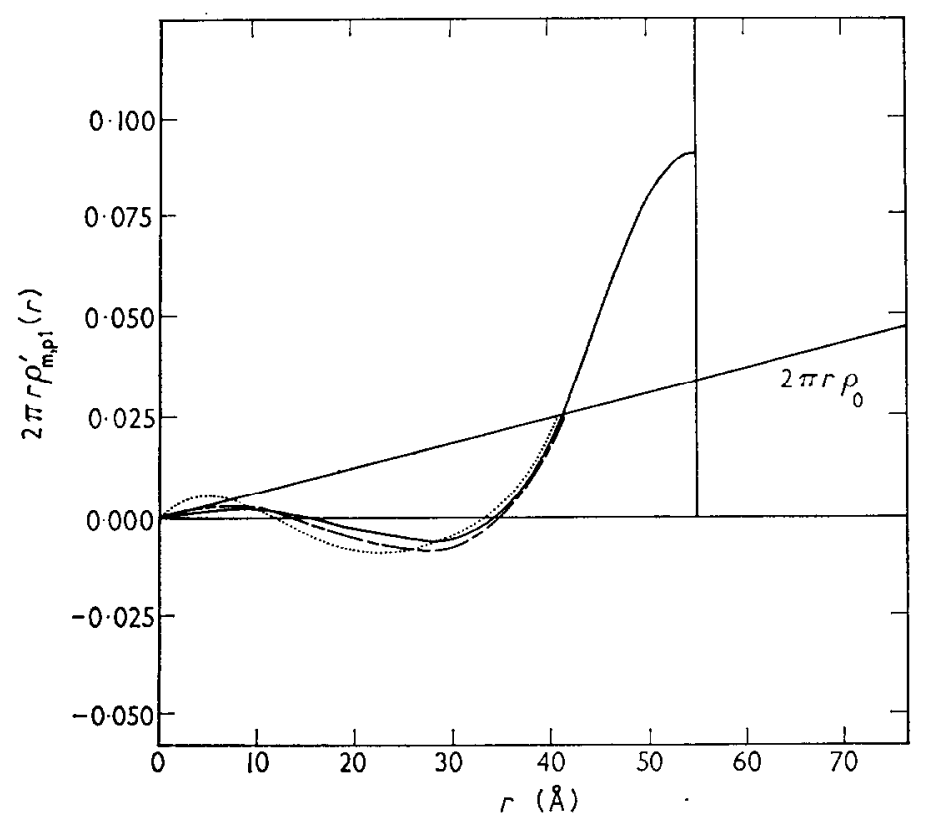

FIG. 10. The planar radial distribution functions for the particles of the wet untreated disk membranes using $f_{\mathrm{pl}}^{2}\left(r^{*}\right)$ and $f_{\mathrm{m}}^{2}\left(r^{*}\right)$ in Fig. 9 compared with that predicted by transform trun. cation alone in the region for $r$ less than the particlo diamoter.

$\ldots \ldots, f_{\mathrm{m}}^{2}\left(r^{*}\right) ;---, f_{\mathrm{pl}}^{2}\left(r^{*}\right) ;-$, transform truncation alone.

This work was aided by a U.S. Public Health Service Grant No. GM-09796. One of us (J. K. B.) is a National Institutes of Health pre-doctoral fellow. 